\title{
Transport and instability in driven two-dimensional magnetohydrodynamic flows
}

\begin{tabular}{|r|l|}
\hline Journal: & Journal of Fluid Mechanics \\
\hline Manuscript ID & JFM-15-S-1108.R2 \\
\hline mss type: & Standard \\
\hline Date Submitted by the Author: & n/a \\
\hline Complete List of Authors: & $\begin{array}{l}\text { Durston, Sam; University of Exeter, Mathematics and Computer Science } \\
\text { Gilbert, Andrew; University of Exeter, Mathematics and Computer Science }\end{array}$ \\
\hline Keyword: & MHD and Electrohydrodynamics, Instability \\
\hline \multicolumn{2}{|l}{} \\
\hline
\end{tabular}

\section{SCHOLARONE $^{\text {m }}$ \\ Manuscripts}




\title{
Transport and instability in driven two-dimensional magnetohydrodynamic flows
}

\author{
Sam Durston and Andrew D. Gilbert \\ Department of Mathematics and Computer Science, \\ College of Engineering, Mathematics and Physical Sciences, \\ University of Exeter, EX4 4QF, U.K.
}

(Received $\mathrm{xx}$; revised $\mathrm{xx}$; accepted $\mathrm{xx}$ )

This paper concerns the generation of large scale flows in forced two-dimensional systems. A Kolmogorov flow with a sinusoidal profile in one direction (driven by a body force) is known to become unstable to a large scale flow in the perpendicular direction at a critical Reynolds number. This can occur in the presence of a beta-effect and has important implications for flows observed in geophysical and astrophysical systems. It has recently been termed 'zonostrophic instability' and studied in a variety of settings, both numerically and analytically.

The goal of the present paper is to determine the effect of magnetic field on such instabilities using the quasi-linear approximation, in which the full fluid system is decoupled into a mean flow and waves of one scale. The waves are driven externally by a given, random body force and move on a fast time scale, while their stress on the mean flow causes this to evolve on a slow time scale. Spatial scale separation between waves and mean flow is also assumed, to allow analytical progress.

The paper first discusses purely hydrodynamic transport of vorticity including zonostrophic instability, the effect of uniform background shear, and calculation of equilibrium profiles in which the effective viscosity varies spatially, through the mean flow. After brief consideration of passive scalar transport or equivalently kinematic magnetic field evolution, the paper then proceeds to study the full MHD system and to determine effective diffusivities and other transport coefficients using a mixture of analytical and numerical methods. This leads to results on the effect of magnetic field, background shear and beta-effect on zonostrophic instability and magnetically driven instabilities.

\section{Introduction}

The aim of this paper is to analyse zonostrophic instability and transport in planar, forced magnetohydrodynamic (MHD) systems. The term zonostrophic turbulence or zonation was introduced in Galperin et al. (2006), referring to the generation of strong zonal flows or jets in two-dimensional turbulent flow driven by an external body force. Srinivasan \& Young (2012) discuss the interaction of mean flows and eddies in forced fluid dynamical systems, and termed the formation of jets 'zonostrophic instability'. Such jets are widely observed in Earth's atmosphere and oceans, in the laboratory and in simulations, and most famously in the banded structures of Jupiter; see, for example, Vallis \& Maltrud (1993), Heimpel, Aurnou \& Wicht (2005), Rotvig \& Jones (2006), Read et al. (2007), Scott \& Polvani (2007), Berloff, Kamenkovich \& Pedlosky (2009) and Galperin et al. (2014). With the identification of this robust fluid dynamical phenomenon goes an ever-expanding literature which we can only outline in what follows.

Instability of a forced fluid flow to large scale zonal flows was first identified in the classic work of Meshalkin \& Sinai (1961) who considered Kolmogorov flow $\boldsymbol{u}=(0, \sin x)$ 
in the plane, with viscous dissipation balanced by an imposed body force. Above a critical Reynolds number $\operatorname{Re}_{c}=2^{-1 / 2}$, the flow becomes unstable to $x$-directed sinusoidal motion, whose scale diverges as $\operatorname{Re}_{c}$ is approached from above. Extensions incorporate the effect of nonlinearity in a multiple scale framework, and a $\beta$-effect corresponding to an imposed background vorticity gradient; see Frisch, Legras \& Villone (1996) and Manfroi \& Young $(1998,2002)$. In the case of zonostrophic instability of a Komogorov flow, the principal effect of nonlinearity is that the zonal flows evolve to increasing spatial scales, a process that is then halted if the parameter $\beta$ is non-zero.

It became apparent that zonostrophic instability is widespread in rotating physical systems, and in laboratory and numerical simulations, and is broadly independent of the way the fluid is driven, for example an imposed body force in a simulation, or convection in a giant planet. With this, further theoretical understanding developed: one route is based on the conservation of potential vorticity (PV) in ideal flows, leading to an understanding of the development of zonal flows in terms of what is known as the PV Phillips effect (Dritschel \& McIntyre 2008): flow evolution naturally leads to inhomogeneities as PV mixing tends to be suppressed in regions of high PV gradient. This negative viscosity or anti-friction effect, maintaining and sharpening gradients, is further studied in high Reynolds number simulations (Dritschel \& Scott 2011; Scott \& Dritschel 2012) and variational principles used to generate possible jet-like structures (Dunkerton \& Scott 2008). In these studies there is the assumption that, although the true system is forced and dissipative these are weak or lower order effects, and it is the ideal evolution or at least the corresponding conservation laws that determine the evolution of zonal flows. We will not discuss these approaches further, noting that in MHD systems conservation of PV is lost, and so these tools are not available in any case.

A second theoretical approach is based on a more detailed modelling of the dynamics, mostly in the framework of the quasi-linear approximation. This incorporates a mean zonal flow (independent of $x$, say) and forced waves (with zero average in $x$ ) whose evolution is linearised about this mean flow. The waves exert mean Reynolds stresses, and with this the mean flow can itself evolve, on a slower time-scale than that of the waves. What is neglected is the interactions of waves giving smaller-scale waves, in other words the beginning of a turbulent cascade. In the context of zonostrophic instability, these ideas and applications are developed in the stochastic structural stability theory of Farrell \& Iouannou (2003, 2006, 2008), Bakas \& Iouannou (2011, 2013, 2014) and Constantinou, Farrell \& Iouannou (2014). Here coupled equations for mean flow and waves, with additional stochastic forcing, are solved numerically in a variety of systems, showing robust jet formation, and the quasi-linear theory validated against direct simulations. Further approximations that bring in higher order corrections, so-called direct statistical simulation, are developed in Tobias, Dagon \& Marston (2011).

To obtain analytical results for zonostrophic instability, Srinivasan \& Young (2012) write the problem for forced flows on a $\beta$-plane in terms of correlation functions in the presence of a weak zonal flow. They then exploit translational symmetry to obtain an exact implicit equation for the instability growth rate, which can be solved numerically to give windows of $y$-wavenumbers $K$ that can be destabilised. Interestingly they find that a destabilising effect linked to the parameter $\beta$ acts as a negative effective hyperviscosity for large scale modes, in other words gives a contribution to the growth rate proportional to $K^{4}$ for wavenumber $K \ll 1$. This is when the system is forced isotropically, whereas for an anisotropic driving, there can be a negative effective viscosity effect, proportional to $K^{2}$ and independent of $\beta$ (as per the original Kolmogorov flow above); see Bakas \& Iouannou (2013), Srinivasan \& Young (2014), and references therein. We stress that these transport effects emerge from the averaged equations for mean, zonal flows. As 
such we are referring only to large scale modes $K \ll 1$ and any $K^{2}$ or $K^{4}$ dispersion relation for growth rate as a function of $K$ must be brought down by further terms as $K$ increases (cf. Sukoriansky, Galperin \& Chekhlov 1999). It is worth noting that in these studies the forced small scale disturbances are damped (by bottom friction or viscosity): they dissipate soon after being created and after depositing any Reynolds stress on the mean flow. In this sense the set-up is diametrically opposite to the theories based on PV conservation in ideal flow. Extending the quasi-linear theory to the case where the waves are forced but only weakly damped raises new challenges. In particular, in the equation for the small scale waves, some quantities converge in the inviscid limit, and others diverge (for example enstrophy). This is studied by Bouchet \& Morita (2010), Bouchet, Nardini \& Tangarife $(2013,2014)$ and Bedrossian \& Masmoudi (2015) for $\beta=0$, and it is established that the quasi-linear model is valid in the limit of zero dissipation for the waves. The formation of zonal flows is further viewed as a problem in pattern formation in Newton, Kim \& Liu (2013) and Parker \& Krommes (2013, 2014), and studied by means of a Ginzburg-Landau equation which incorporates further nonlinear terms.

Our principal focus is the MHD problem: how are processes of zonostrophic instability on a $\beta$-plane modified in the presence of a magnetic field (taken to point in the zonal direction)? Although our focus is not on applications, we mention that in the Earth's core as well as astrophysical bodies, magnetic fields interact with turbulence, rotation and shear. An example is the solar tachocline, where the interaction of magnetic field, shear and convection remains poorly understood (see, e.g., Hughes, Rosner \& Weiss 2007), while dynamo action in the presence of zonal flows is studied in Aubert (2005) and Yadav et al. (2015), and references therein. There are few studies of the role of magnetic field in zonostrophic instability: however Diamond et al. (2007) argue that in turbulent regimes magnetic fields will have the effect of suppressing any inverse cascade of energy that would result in zonal flows, while Tobias, Hughes \& Diamond (2012) find computationally that even weak magnetic field $B_{0}$ can suppress the instability with a threshold $B_{0}^{2} \propto \eta$, the magnetic diffusivity. A similar suppression is found in the presence of ambient shear $\Omega_{0}$ by Srinivasan \& Young (2014) and Hsu \& Diamond (2015). Although there has been little work on zonal flow generation in the presence of magnetic fields, there have been many studies on the modification of transport processes and subsequent generation of magnetic fields, particularly by dynamo action in three dimensions (e.g., Zheligovsky 2011), which we will not review here. Very relevant to us, however, are studies of the modification of transport effects in forced two-dimensional flows threaded by magnetic fields. Chechkin (1999) determines transport effects in terms of the correlation properties of random small scale fields, and shows that the effective viscosity can change sign. In a series of papers, Leprovost \& Kim (2008a,b, 2009) determine effective magnetic diffusivity and viscosity in the limits of strong and weak magnetic field and imposed shear flow; also identified is an additional coupling term in the averaged Navier-Stokes equation related to the gradient of the zonal magnetic field. Keating \& Diamond (2008) determine transport effects for turbulent MHD flow on a $\beta$-plane using weak turbulence theory and find that the combination of these two effects can lead to an increase in effective magnetic diffusivity.

Our goal is to present a study of transport and zonostrophic instability in MHD flow on a $\beta$-plane, in which we go from the governing equations and forcing, through calculations of transport coefficients, to plots of instability thresholds and equilibrium profiles. We also incorporate ambient shear into the system we study, given by a parameter $\Omega_{0}$. We work in the quasi-linear framework and additionally assume a scale separation between the waves and the zonal flow, to allow analytical progress. Although our study builds on earlier work it is distinguished by, firstly a framework that easily allows different forms of molecular 
diffusive/viscous terms, although we always use the Laplacian in our results (rather than molecular hyperdiffusion/hyperviscosity), and secondly in the range of physical effects considered, ultimately parameterised by $B_{0}, \Omega_{0}, \beta$ and a diffusion/viscosity parameter $\lambda$. With this go two caveats: we do not attempt to verify the results by means of full simulations in this paper, and we indeed recognise that quasi-linear theory may in many regimes be only a qualitative guide to the behaviour realised in the full nonlinear fluid system.

The paper is structured as follows: in section 1 we set out the governing equations and the quasi-linear system we will study. We take the driving of the flow, in the $(x, y)$ plane, to be as basic as possible, a periodic excitation of a wave proportional to $e^{i m x}$ with a random phase; note that this driving is anisotropic, which has implications for the transport effects that emerge. In section 2 we focus on the purely hydrodynamic problem, and determine the feedback on the mean flow from a wave, both numerically and analytically. We identify that zonostrophic instability can occur at large scales through a negative effective viscosity term that emerges in the equation for large scale zonal flow. We also determine how the instability is suppressed by an ambient shear $\Omega_{0}$ and solve for equilibrium profiles, in which a steady large scale flow exhibiting a vorticity step is maintained as a result of the varying effective viscosity. We then incorporate a passive scalar field in section 4 and determine the corresponding modifications to passive scalar diffusion and profiles. In these sections we make contact with related studies Srinivasan \& Young $(2012,2014)$ and Hsu \& Diamond (2015) with reference to negative effective viscosity effects, ambient shear and passive scalar evolution, as detailed in the text. Section 5 is devoted to the MHD problem, introducing the Lorentz force to the framework developed in earlier sections. The problem becomes much more complicated, but with a mixture of analytical development and numerical evaluation, we produce thresholds for instabilities in the presence of magnetic fields, shear, and $\beta$-effect. Finally section 6 offers conclusions and future directions.

\section{Governing equations}

Our starting point is two-dimensional MHD flow on a $\beta$-plane, with governing equations

$$
\begin{aligned}
\partial_{t} \omega+J(\omega, \psi) & =J(j, a)+\beta \partial_{x} \psi+\nu \nabla^{2} \omega+s, \\
\partial_{t} a+J(a, \psi) & =\eta \nabla^{2} a, \\
\partial_{t} \sigma+J(\sigma, \psi) & =\kappa \nabla^{2} \sigma .
\end{aligned}
$$

Here $\psi$ is the stream function and $a$ the vector potential, with flow $\boldsymbol{u}=\left(\partial_{y} \psi,-\partial_{x} \psi\right)$ and magnetic field $\boldsymbol{b}=\left(\partial_{y} a,-\partial_{x} a\right)$ (measured in units of velocity). We have included also a passive scalar $\sigma$, which obeys the same equation as the vector potential but with no Lorentz feedback term. The vorticity and current are given by

$$
\omega=-\nabla^{2} \psi, \quad j=-\nabla^{2} a .
$$

All fields are functions of $(x, y, t)$ and $J$ denotes the usual Jacobian with respect to $(x, y)$ coordinates. In (2.1) the flow is driven by the source term $s$, the curl of a body force, which generates vorticity in the fluid, and which we prescribe below.

\subsection{Non-dimensionalisation}

To identify the parameters involved, it is helpful to write the governing equations in a non-dimensional form. The external input to the system is the vorticity source term $s(x, y, t)$ and we take it to have magnitude $\mathcal{S}$, and act on a spatial scale $\mathcal{L}$. We choose to 


\begin{tabular}{lccllccc}
\hline quantity & mean & fluctuating & quantity & mean & fluctuating \\
& & & & & & & \\
vorticity & $\Omega=-U^{\prime}$ & $\omega$ & $\zeta$ & current & $J=-B^{\prime}$ & $j$ & $\gamma$ \\
zonal velocity & $U=\Psi^{\prime}$ & & & magnetic field & $B=A^{\prime}$ & & \\
stream function & $\Psi$ & $\psi$ & $\phi$ & vector potential & $A$ & $a$ & $\alpha$ \\
passive scalar & $\Sigma$ & $\sigma$ & $c$ & & & &
\end{tabular}

TABLE 1. Mean and fluctuating fields (the second of each pair has had the pure advection term $e^{-i m U(y) t}$ removed).

use the length scale $\mathcal{L}$ and the time scale $\mathcal{T} \equiv \mathcal{S}^{-1 / 2}$ to non-dimensionalise the problem, rescaling quantities such as

$\omega=\omega^{\dagger} / \mathcal{T}, \quad a=a^{\dagger} \mathcal{L}^{2} / \mathcal{T}, \quad \beta=\beta^{\dagger} / \mathcal{L} \mathcal{T}, \quad \nu=\nu^{\dagger} \mathcal{L}^{2} / \mathcal{T}, \quad \eta=\eta^{\dagger} \mathcal{L}^{2} / \mathcal{T}, \quad \kappa=\kappa^{\dagger} \mathcal{L}^{2} / \mathcal{T}$.

This recovers equations (2.1-2.4) in a dimensionless form, adorned with daggers. The system is then characterised by the dimensionless parameters, the Grashof number Gr and the source Rhines number $\mathrm{Rh}_{\mathrm{s}}$, given by

$$
\mathrm{Gr}^{-1 / 2} \equiv \nu^{\dagger}=\nu / \mathcal{L}^{2} \sqrt{\mathcal{S}}, \quad \mathrm{Rh}_{\mathrm{s}}^{-1} \equiv \beta^{\dagger}=\beta \mathcal{L} / \sqrt{\mathcal{S}}
$$

as well as Prandtl and magnetic Prandtl numbers giving the ratios of the appropriate diffusivities, and the functional form of the source term $s$. Note that $\mathrm{Rh}_{\mathrm{s}}$ is here based on the source strength rather than on the actual flow velocities realised, whereas the usual Rhines number $\mathrm{Rh}=\mathcal{U} / \beta \mathcal{L}^{2}$, like the Reynolds number $\mathrm{Re}=\mathcal{L U} / \nu$, would be a diagnostic depending on the flow velocities $\mathcal{U}$ that emerge in the forced system (Rhines 1975).

As a key simplification in this study we will take all the diffusivities to be equal,

$$
\nu^{\dagger}=\eta^{\dagger}=\kappa^{\dagger} \equiv \lambda^{\dagger}, \quad \text { say, }
$$

so the Prandtl numbers are unity, and we use the neutral quantity $\lambda^{\dagger}$ to denote any diffusivity or viscosity in what follows. This is both to make analytical progress and to reduce the number of parameters involved, making our study more manageable. For ease of notation it is also convenient to use the dimensionless parameters $\left\{\lambda^{\dagger}, \beta^{\dagger}\right\}$ in what follows rather than $\left\{\mathrm{Gr}, \mathrm{Rh}_{\mathrm{s}}\right\}$. From now on we will also drop the daggers on nondimensional quantities and so the key parameters at the outset are simply $\{\lambda, \beta\}$, with the source $s$ taken of length scale and magnitude of order unity.

\subsection{Quasi-linear approximation}

We adopt the quasi-linear approximation in which we take a mean or background flow and magnetic field independent of $x$, namely

$\omega=\Omega(y, t), \quad u_{1}=U(y, t), \quad \psi=\Psi(y, t), \quad j=J(y, t), \quad b_{1}=B(y, t), \quad a=A(y, t), \quad \sigma=\Sigma(y, t)$,

and assume these are quasi-steady, varying on a longer time scale than that of the fluctuating fields, namely those fields with a non-trivial $x$-dependence. We need then to define the source term $s$ which creates disturbances, evolving according to linear dynamics, linearised about the background profile (2.8). Take first the specific case of a delta-function (in time) source of vorticity that is a single Fourier mode in $x$,

$$
s=\delta(t) e^{i m x}+\text { c.c. } \quad(m>0) .
$$


In the quasi-linear approximation we break fields into mean and fluctuating components as summarised in table 1 ,

$$
(\omega, \psi, j, a, \sigma) \rightarrow(\Omega, \Psi, J, A, \Sigma)+(\omega, \psi, j, a, \sigma)(y, t) e^{i m x}+\text { c.c. }+\cdots,
$$

and retain only the leading harmonics shown. We refer to an evolving $e^{i m x}$ disturbance as a wave (even in regimes where it may be heavily damped), and this obeys linear equations

$$
\begin{aligned}
& \partial_{t} \omega+i m U \omega-i m B j=i m\left(\beta+\Omega^{\prime}\right) \psi-i m J^{\prime} a+\lambda \Delta \omega, \\
& \partial_{t} a+i m U a-i m B \psi=\lambda \Delta a, \\
& \partial_{t} \sigma+i m U \sigma-i m \Sigma^{\prime} \psi=\lambda \Delta \sigma, \\
& \omega=-\Delta \psi, \quad j=-\Delta a, \quad \Delta \equiv-m^{2}+\partial_{y}^{2}
\end{aligned}
$$

with initial conditions from (2.9),

$$
\omega(y, 0)=1, \quad \psi(y, 0)=m^{-2}, \quad j(y, 0)=a(y, 0)=\sigma(y, 0)=0 .
$$

A prime here denotes the $y$-derivative of any mean field, these being related as shown in table 1.

The feedback on the mean fields in (2.10) from the waves via the quadratic terms in $(2.1-2.3)$ is retained, and this can be written in terms of fluxes

$$
\begin{aligned}
& F_{\mathrm{R}}(y, t)=i m\left(\psi^{*} \omega-\psi \omega^{*}\right), \quad F_{\mathrm{L}}(y, t)=i m\left(a^{*} j-a j^{*}\right), \\
& F_{\mathrm{M}}(y, t)=i m\left(\psi^{*} a-\psi a^{*}\right), \quad F_{\mathrm{P}}(y, t)=i m\left(\psi^{*} \sigma-\psi \sigma^{*}\right),
\end{aligned}
$$

from Reynolds and Lorentz stresses in (2.16), and from advection of vector potential and scalar fluctuations in (2.17). Over the life-time of a wave, the feedback on the mean flow from any one of these fluxes is given by the integral

$$
\mathcal{F}_{\mathrm{Z}}(y)=\int_{0}^{\infty} F_{\mathrm{Z}}(y, t) d t
$$

Here and elsewhere we find it convenient to use $\mathrm{Z}$ as a place-holder: it can be R, L, M or $\mathrm{P}$ as in $(2.16,2.17)$, or involve further symbols later on.

We have the equations (2.11-2.15) for a single wave launched at $t=0$ and the feedback $(2.16-2.18)$ on the mean fields as the wave evolves and dissipates. As this effect must be weak in any quasi-linear set-up, we now incorporate a stream of such waves, replacing (2.9) by the renewing source

$$
s=\sum_{j} \delta(t-j) e^{i m x+i \mu_{j}}+\text { c.c. } \quad(m>0),
$$

in which at each unit of time vorticity is introduced into the flow with random, independent phases $\mu_{j}$ uniformly distributed over $[-\pi, \pi]$. Given the random phases, in a time average (or ensemble average) all waves contribute independently to the quadratic fluxes. The equations for the mean fields then become

$$
\begin{aligned}
& \partial_{t} \Omega+\partial_{y} \mathcal{F}_{\mathrm{K}}=\lambda \partial_{y}^{2} \Omega, \\
& \partial_{t} A+\partial_{y} \mathcal{F}_{\mathrm{M}}=\lambda \partial_{y}^{2} A, \\
& \partial_{t} \Sigma+\partial_{y} \mathcal{F}_{\mathrm{P}}=\lambda \partial_{y}^{2} \Sigma,
\end{aligned}
$$

where it is convenient to combine Reynolds and Lorentz terms via

$$
F_{\mathrm{K}}=F_{\mathrm{R}}-F_{\mathrm{L}}, \quad \mathcal{F}_{\mathrm{K}}=\mathcal{F}_{\mathrm{R}}-\mathcal{F}_{\mathrm{L}} .
$$


Note that the term $\mathcal{F}_{\mathrm{K}}$ would also appear in a mean momentum equation, as per (3.18) below, but we usually prefer to remain within a vorticity-stream function formulation.

\subsection{Summary and comments}

The quasi-linear system that we will consider is well-established in the literature and consists of solving (2.11-2.15) for general mean profiles $U, B$ and $\Sigma$, and then determining the feedback on each mean field through the fluxes (2.16-2.18) that feed into (2.20-2.22). Several comments are in order:

(i) Given the renewing source (2.19), the equations (2.20-2.21) for the mean fields are only valid on time scales $t \gg 1$, that is over many waves launched into the flow. Furthermore the quasi-linear approximation (as we use it) keeps the mean fields $U$ and $B$ steady while we solve (2.11-2.15) and compute the integrated fluxes in (2.18). There is thus a key consistency condition, that the time scale of evolution of the mean fields in $(2.20-2.22)$ be greater than that over which the waves contribute to the integrated fluxes (2.18), as stressed by Bouchet \& Morita (2010). This consistency condition needs to be assessed as any quasi-linear model is developed.

(ii) One of the features of this study is keeping the source term (2.19) as tractable as possible without too much loss of generality. Regarding this, we note that we could have introduced a more general time dependence, for example involving a stationary random process with a given correlation function (decaying on a time scale shorter than that of the evolution of the mean fields), for example delta-correlated. However doing so would increase complexity for us without adding greatly to generality. In fact we can note that the problem as formulated in (2.11-2.15) gives the Green's function for waves, and the effect of a more general time dependence of the source $s$ could thus be obtained by integration.

(iii) Likewise we have considered a single source mode with wave number $m$ in (2.19); we treat $m$ as a parameter (though strictly it could be scaled to $m=1$ in our nondimensionalisation). More generally we could consider modes of the form $e^{i m x+i n y}$ and arbitrary sums of such modes, for example forming an isotropic or ring forcing as used in many other studies. However because of shearing in gradients $U^{\prime}$ of the mean flow, a more general mode $e^{i m x+i n y}$ simply corresponds to a shift in time of the mode $e^{i m x}$ and so our analysis could be extended, creating additional complexity in integrating over angles but without any fundamental changes. We should remark though that our forcing is anisotropic, in contrast to some other studies, and this is known to enhance instabilities leading to zonal flows, by selecting a preferred direction in the system, as discussed in Srinivasan \& Young (2012) and Bakas \& Iouannou (2013).

(iv) We have not discussed the strength of the background flow $U$, scalar field $\Sigma$ or magnetic field $B$. In fact the status of these is rather different. A background flow $U$ can grow from a seed through zonostrophic instability, and so we cannot set its scale at the outset. For passive scalar transport, the magnitude of the background gradient $\Sigma^{\prime}$ is a constant that can be set arbitrarily. The strength of a mean magnetic field (that is, $B$ averaged over the $y$-coordinate) is another constant that must be set initially and will introduce a further parameter into the problem. Note that given our present nondimensionalisation (2.5), magnetic field strength $B$ is measured relative to the velocity strength, corresponding to an inverse magnetic Mach number.

(v) Our primary focus will be on $\nabla^{2}$ dissipation (we refer to this as molecular dissipation) in the development from (2.1-2.3), but we will below remark briefly on more 
general forms of dissipation, using $-\lambda_{r}\left(-\nabla^{2}\right)^{r} \omega$ in (2.1) and likewise for $a$ and $\sigma$. The case $r=0$ is bottom drag, $r \geqslant 2$ hyperviscosity (or hyperdiffusion) and $r=1, \lambda_{1} \equiv \lambda$ standard viscous dissipation. We stress that these are the 'molecular' effects - in other words terms that we introduce into the governing equations, to be distinguished from the 'effective' transport effects, for example $\nu_{\text {eff }}, \kappa_{\text {eff }}$, that emerge from our analysis below.

\section{Hydrodynamic transport}

Although the purely hydrodynamic problem has been well explored in the literature, it is naturally a starting point for the MHD problem. In order to set the scene, give a point of comparison, and establish scalings for the MHD problem, we sketch this case. We need to solve for waves on a given profile $U$, from (2.11),

$$
\partial_{t} \omega+i m U \omega=i m\left(\beta+\Omega^{\prime}\right) \psi+\lambda \Delta \omega, \quad \omega=-\Delta \psi .
$$

\subsection{Multiple scale formulation}

We first apply a transformation to absorb the advective term,

$$
\omega(y, t)=e^{-i m U(y) t} \zeta(y, t), \quad \psi(y, t)=e^{-i m U(y) t} \phi(y, t)
$$

and obtain

$$
\partial_{t} \zeta=i m\left(\beta+\Omega^{\prime}\right) \phi+\lambda \mathcal{L} \zeta, \quad \zeta=-\mathcal{L} \phi,
$$

with $\mathcal{L}$ denoting the Laplacian operator

$$
\zeta=-\mathcal{L} \phi=m^{2}\left(1+\Omega^{2} t^{2}\right) \phi-i m \Omega^{\prime} t \phi-2 i m \Omega t \partial_{y} \phi-\partial_{y}^{2} \phi .
$$

The flux (2.16) may then be written as

$$
F_{\mathrm{R}}=\partial_{y}\left[2 m^{2} \Omega t|\phi|^{2}-i m\left(\phi^{*} \partial_{y} \phi-\phi \partial_{y} \phi^{*}\right)\right] .
$$

Note that the leading order term in $\zeta$ from (3.4), which is a purely real multiple $m^{2}(1+$ $\left.\Omega^{2} t^{2}\right)$ of $\phi$, does not contribute to the flux $F_{\mathrm{R}}$. This absence of a phase shift between the two fields means that a vorticity disturbance on an exactly linear shear flow $\Omega=$ const. cannot contribute a mean Reynolds stress: it is higher order terms that need to be included, involving gradients of $\Omega$.

The development so far is exact (within the quasi-linear approximation) and to make further progress we take the case when the mean or background flow varies on a large scale $Y$, setting

$$
Y=\varepsilon y, \quad T=\varepsilon t, \quad U(y) \rightarrow U(Y), \quad \Omega(y) \rightarrow \varepsilon \Omega(Y),
$$

with $\varepsilon \ll 1$. We now write $\Omega=-U^{\prime}$, using the prime to denote a $Y$-derivative of any background field. Here and elsewhere we suppress the (slow) time dependence of the background field, governed by (3.12) below. (Note that the correlation function approach of Srinivasan \& Young (2012) does not assume scale separation.)

We now adopt the scalings

$$
\begin{array}{ll}
\omega \rightarrow \varepsilon^{3 / 2} \omega(Y, T), \quad \psi \rightarrow \varepsilon^{3 / 2} \psi(Y, T), \quad \zeta \rightarrow \varepsilon^{3 / 2} \zeta(Y, T), \quad \phi \rightarrow \varepsilon^{3 / 2} \phi(Y, T), \\
F_{\mathrm{R}} \rightarrow \varepsilon^{4} F_{\mathrm{R}}(Y, T) \quad \mathcal{F}_{\mathrm{R}} \rightarrow \varepsilon^{3} \mathcal{F}_{\mathrm{R}}(Y), \quad \lambda \rightarrow \varepsilon \lambda, \quad \beta \rightarrow \varepsilon \beta .
\end{array}
$$

These give for the wave evolution on the $T$ time scale,

$$
\begin{aligned}
& \partial_{T} \zeta=i m\left(\beta+\varepsilon \Omega^{\prime}\right) \phi+\lambda \mathcal{L} \zeta, \\
& \zeta=-\mathcal{L} \phi=m^{2}\left(1+\Omega^{2} T^{2}\right) \phi-i \varepsilon m \Omega^{\prime} T \phi-2 i \varepsilon m \Omega T \partial_{Y} \phi-\varepsilon^{2} \partial_{Y}^{2} \phi,
\end{aligned}
$$


with

$$
F_{\mathrm{R}}=\partial_{Y}\left[2 m^{2} \Omega T|\phi|^{2}-i \varepsilon m\left(\phi^{*} \partial_{Y} \phi-\phi \partial_{Y} \phi^{*}\right)\right] .
$$

The equation (2.20) for mean fields takes the form

$$
\partial_{\mathcal{T}} \Omega+\partial_{Y} \mathcal{F}_{\mathrm{R}}=\lambda \partial_{Y}^{2} \Omega,
$$

and these fields develop on the long time scale $\mathcal{T}=\varepsilon^{2} T$, where as a consequence of the new scalings in (3.8), the equation

$$
\mathcal{F}_{\mathrm{Z}}=\int_{0}^{\infty} F_{\mathrm{Z}} d T
$$

now replaces (2.18) for any label Z.

Equations (3.9-3.13) are the ones we wish to expand and study asymptotically for small $\varepsilon$. The rationale for the above choice of scaling in $(3.7,3.8)$ is to retain as many terms at leading order as possible in (3.9-3.13). Note that from (3.7), the strength of the waves, and so the strength of the original driving force, is being reduced as $\varepsilon \rightarrow 0$, to balance the viscous term in (3.12) for the mean fields. For more general dissipation the scalings would be as above except for

$$
\mathcal{T}=\varepsilon^{2 r} T, \quad \zeta \rightarrow \varepsilon^{r+1 / 2} \zeta, \quad \phi \rightarrow \varepsilon^{r+1 / 2} \phi, \quad F_{\mathrm{R}} \rightarrow \varepsilon^{2 r+2} F_{\mathrm{R}} \quad \mathcal{F}_{\mathrm{R}} \rightarrow \varepsilon^{2 r+1} \mathcal{F}_{\mathrm{R}}
$$

and give rise to the same set of equations with only modifications to the definition of $\mathcal{L}$ in (3.10). For $r=0$ (linear drag) there is no separation of scales between $\mathcal{T}$ and $T$ and so the consistency condition discussed in the first comment of section 2.3 would not be satisfied: mean flow and waves would be damped on the same time scale (at least, unless some further limit were taken, or perhaps some combination of damping effects employed).

\subsection{Numerical solution}

Before undertaking further analysis, it is worth gaining some intuition by simulating the system we have so far for the linear waves $\omega(Y, T), \psi(Y, T)$. The system may be written as

$$
\begin{aligned}
& \partial_{T} \omega+i m \varepsilon^{-1} U \omega=i m\left(\beta+\varepsilon \Omega^{\prime}\right) \psi+\lambda\left(\varepsilon^{2} \partial_{Y}^{2}-m^{2}\right) \omega, \\
& -\omega=\left(\varepsilon^{2} \partial_{Y}^{2}-m^{2}\right) \psi,
\end{aligned}
$$

equivalent to $(3.9,3.10)$, with here

$$
F_{\mathrm{R}}(y, t)=i m \varepsilon^{-1}\left(\psi^{*} \omega-\psi \omega^{*}\right) .
$$

A run with $\beta=0, \varepsilon=0.1$ and $U=\cos Y$ is shown in figure 1: panels (a,b) show the real part of the vorticity $\omega$ and stream function $\psi$ respectively, in the $(T, Y)$-plane. We have taken zero viscosity $\lambda=0$, otherwise we would simply see a damping effect. Away from the maxima of $|U|$ at $Y=0, \pm \pi$ (where $\Omega=0$ ) the vorticity gains finer scales and the stream function is suppressed. The flux $F_{\mathrm{R}}$ is shown in panel (c) and develops (red) peaks and (blue) troughs for large $T$.

The short time evolution of flux $F_{\mathrm{R}}(Y, T)$ is shown in panel (d) for $T=0.1,0.2, \ldots, 1$ (solid), going from innermost (smoothest) curve to outermost. Panel (d) contains key information as we can write from (3.12) for the mean velocity

$$
\partial_{\mathcal{T}} U=\mathcal{F}_{\mathrm{R}}+\lambda \partial_{Y}^{2} U
$$

and here $U=\cos Y$ is shown dashed in figure 1(d). Thus the short time effect of the flux 
(a)

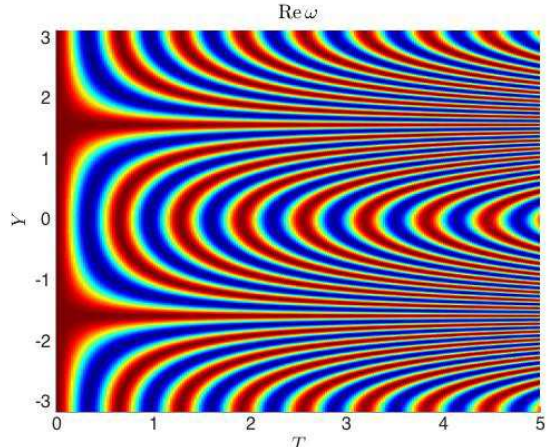

$F_{R}$

(c)

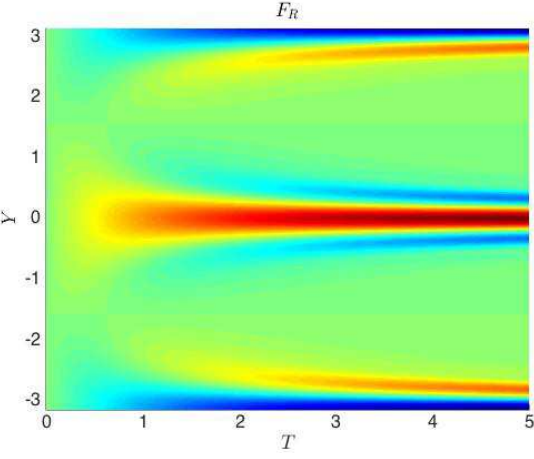

(b)

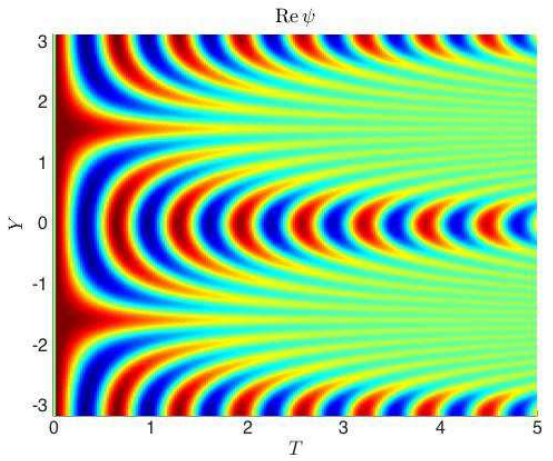

(d)

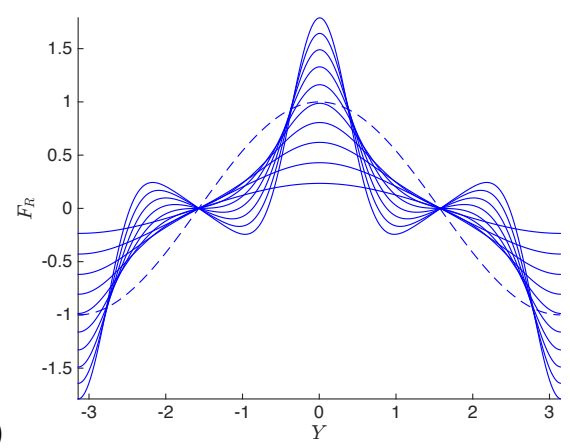

FiguRE 1. Simulation of waves with $\beta=0, m=1, \varepsilon=0.1, \lambda=0$ and $U=\cos Y$. In (a) $\operatorname{Re} \omega$, (b) $\operatorname{Re} \psi$ and (c) $F_{R}$ are plotted in the $(T, Y)$-plane. In (d) $F_{R}(Y, T)$ is shown as a function of $Y$ for $T=0.1,0.2, \ldots, 1$ (solid, innermost to ouermost curve), with $U(Y)$ (dashed).

$F_{\mathrm{R}}$ (as it contributes to $\mathcal{F}_{\mathrm{R}}$ ) is to reinforce the original flow $U$, which is precisely the effect leading to zonostrophic instability. If there is sufficient viscous damping, then only the short time feedback is relevant and below we will recover this effect, which naturally is well documented in the literature (e.g. Srinivasan \& Young 2012).

For greater times, the feedback from the flux $F_{\mathrm{R}}$ becomes more oscillatory in $Y$, changing sign in places with respect to the original $U=\cos Y$ profile, as seen in figure $1(\mathrm{c}, \mathrm{d})$. This is investigated further below, but there is an indication that the effect of the feedback is to sharpen any pre-existing profile, increasing the flow where $\Omega=-U^{\prime}=0$ and flattening the flow profile where $U=0$ and $|\Omega|$ is maximal. Note that a tendency to sharpen shear profiles is found in weakly damped vortex dynamics simulations by Dritschel \& McIntyre (2008), and by Kim \& MacGregor (2003) in a study of gravity waves in stratified fluid, together with a bifurcation to an oscillatory state.

\subsection{Multiple scale expansion}

We now return to the development in section 3.1 and approximate. We expand quantities in powers of $\varepsilon$, for example,

$$
\zeta=\zeta_{0}+\varepsilon \zeta_{1}+\cdots, \quad \phi=\phi_{0}+\varepsilon \phi_{1}+\cdots,
$$

and the leading order equations $(3.9,3.10)$ take the form

$$
\partial_{T} \zeta_{0}=i m \beta \phi_{0}-\lambda m^{2}\left(1+\Omega^{2} T^{2}\right) \zeta_{0}, \quad \zeta_{0}=m^{2}\left(1+\Omega^{2} T^{2}\right) \phi_{0} .
$$

The accelerated decay through the $T^{3}$ term in the exponential is known as the sheardiffuse mechanism (Bernoff \& Lingevitch 1994) responsible for flux expulsion in kinematic 
magnetic field evolution (Weiss 1966). This gives a first order ODE with respect to $T$, with the solution

$$
\zeta_{0}=\exp \left[i m^{-1} \beta \Omega^{-1} \tan ^{-1} \Omega T\right] \exp \left[-m^{2} \lambda\left(T+\frac{1}{3} \Omega^{2} T^{3}\right)\right] .
$$

The expansion gives us functions of $\Omega T$ rather than $T$ and so it is convenient call this $\tau$ and to try to reduce the number of parameters involved. We set

$$
\tau=\Omega T, \quad \hat{\beta}=\beta / m \Omega, \quad \hat{\lambda}=m^{2} \lambda / \Omega, \quad D(\tau, \hat{\lambda})=\exp \left[-\hat{\lambda}\left(\tau+\frac{1}{3} \tau^{3}\right)\right],
$$

in terms of which we have the more compact form

$$
\zeta_{0}=\exp \left[i \hat{\beta} \tan ^{-1} \tau\right] D
$$

We will for simplicity and without loss of generality consider points $Y$ in the profile where $\Omega>0$, so that $\tau \in[0, \infty]$; the final results are nonetheless correct also for $\Omega<0$. Behaviour at points where $\Omega=0$ can be established by letting $\Omega \rightarrow 0$; although this need not detain us here, we make some further comments and qualifications in appendix A.

We then obtain the leading order flux from (3.11) as a function of time,

$$
F_{\mathrm{R}}=2 m^{-2} \partial_{Y}\left[\tau\left(1+\tau^{2}\right)^{-2} D^{2}\right] \equiv-m^{-2} \Omega^{\prime} \Omega^{-1} D^{2} f_{\mathrm{R}}
$$

(we have simply dropped the term of order $\varepsilon$ in (3.11), but not further complicated the notation) with

$$
f_{\mathrm{R}}(\tau, \hat{\lambda})=-2 \tau\left(1+\tau^{2}\right)^{-2}\left[\left(1-3 \tau^{2}\right)\left(1+\tau^{2}\right)^{-1}-\frac{4}{3} \hat{\lambda} \tau^{3}\right] .
$$

The leading order integral may be written as

$$
\mathcal{F}_{\mathrm{R}}=-\nu_{\text {eff }} \Omega^{\prime}, \quad \nu_{\text {eff }}=m^{-2} \Omega^{-2} h_{\mathrm{R}},
$$

where we set, for any label Z,

$$
h_{\mathrm{Z}}=\int_{0}^{\infty} D^{2} f_{\mathrm{Z}} d \tau .
$$

Note that $h_{\mathrm{R}}=h_{\mathrm{R}}(\hat{\lambda})$ only, and in fact $\hat{\beta}$ has dropped out of the calculations. The large scale equation is now

$$
\partial_{\mathcal{T}} \Omega=\partial_{Y}\left[\nu_{\mathrm{eff}} \partial_{Y} \Omega\right]+\lambda \partial_{Y}^{2} \Omega
$$

with an effective viscosity $\nu_{\text {eff }}(\Omega, \lambda)$ identified and arising from the externally forced waves. This term emerges from the two-scale analysis with the expected form, in other words giving transport of the large scale vorticity field $\Omega(Y, \mathcal{T})$. Note however that although the underlying molecular viscosity $\lambda$ is always positive and so dissipative in effect, given we have averaged over the vorticity equation with an imposed body force present, there is no reason why the effective (or 'renormalised') viscosity $\nu_{\text {eff }}$ need be positive, and generally it is not, this being the origin of zonostrophic instability in this system.

To summarise, equation (3.24) gives the instantaneous feedback $F_{\mathrm{R}}$ from the wave to the mean flow, coded in the function $f_{\mathrm{R}}(\tau, \hat{\lambda})$ in $(3.25)$ with the damping factor $D^{2}$. The integrated effect is found in $h_{\mathrm{R}}(\hat{\lambda})$ and gives the effective viscosity in (3.26); the parameter $\hat{\beta}$ plays no role at this order (cf. Srinivasan \& Young 2012, 2014). Figure 2(a) shows $D^{2} f_{\mathrm{R}}$ as a function of $\tau$ in the inviscid limit $\hat{\lambda}=0$ and for increasing values of $\hat{\lambda}$. Focusing on the inviscid case (outermost curve) it is worth noting that the feedback changes sign at $\tau=3^{-1 / 2}$. The effect of increasing the damping $\hat{\lambda}>0$ (inner curves), is 
(a)

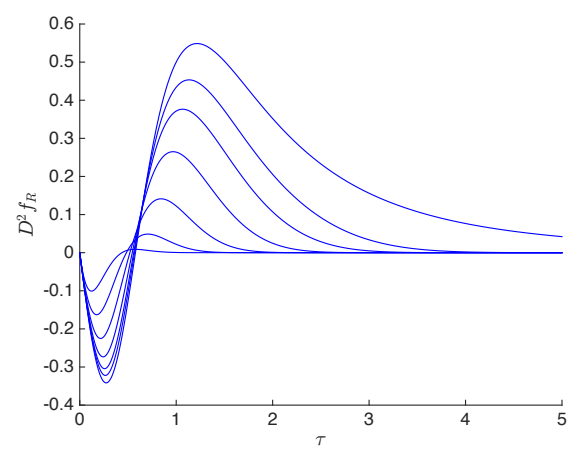

(b)

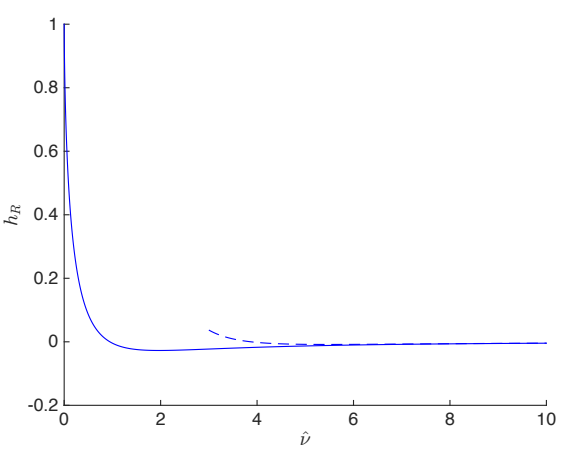

Figure 2. (a) Plot of $D^{2} f_{\mathrm{R}}$ as a function of $\tau$ for $\hat{\lambda}=0,0.1,0.2,0.4, \ldots, 3.2$ (from outermost to innermost curves). (b) Plot of $h_{\mathrm{R}}$ as a function of $\hat{\lambda}$ (solid) and the approximation (3.29) (dashed).

primarily to suppress $f_{\mathrm{R}}$ at ever earlier times. Figure $2(\mathrm{~b})$ shows $h_{\mathrm{R}}(\hat{\lambda})$ which is the total integrated flux. We have $h_{\mathrm{R}}(0)=1$ while for small $\hat{\lambda}$ the effective viscosity is positive, corresponding to the damping of the mean flow. At $\hat{\lambda} \simeq 0.95, h_{\mathrm{R}}$ changes sign and for larger $\hat{\lambda}$ it is negative and so destabilising. For large $\hat{\lambda}$ the integral (3.27) is effectively cut off at times $\tau=O\left(\hat{\lambda}^{-1}\right)$ and we can expand the functions $D$ and $f_{\mathrm{R}}$ as power series in $\tau$ giving

$$
h_{\mathrm{R}}(\hat{\lambda})=-\frac{1}{2} \hat{\lambda}^{-2}+\frac{15}{2} \hat{\lambda}^{-4}+\cdots, \quad \nu_{\mathrm{eff}}=-\frac{1}{2} m^{-6} \lambda^{-2}+\frac{15}{2} m^{-10} \Omega^{2} \lambda^{-4}+\cdots \quad(\hat{\lambda} \gg 1),
$$

also shown on figure 2(b) (dashed).

Let us now relate this detailed calculation back to the bigger picture, for example as in the simulations in figure 1. For good scale separation $\varepsilon \ll 1$, at each $Y$ the flow is locally shearing as given by the value of $\Omega(Y)$. The subsequent evolution of a wave is determined by the key parameter $\hat{\lambda}$ and occurs on a time scale $\tau$; see (3.22). The evolution on the $\tau$ time scale is damped by the factor $D$, which occurs at $\tau=O\left(\hat{\lambda}^{-1}\right)$ for large $\hat{\lambda}$, or at $\tau=O\left(\hat{\lambda}^{-1 / 3}\right)$ for small $\hat{\lambda}$, by the shear-diffuse mechanism. For small $\varepsilon$, this is all that is needed to recreate the evolution of $F_{\mathrm{R}}$ in figure 1(c,d), and for each $Y$ good agreement can be shown between the evolution in time $T$ in this figure, and in the analysis above based on the local ODE (3.20).

Note that in a profile with varying $\Omega$, the local parameter $\hat{\lambda}$ will also vary. Taking $\Omega$ bounded, if $\lambda \gg 1$ then the waves are always in a viscous regime $\hat{\lambda} \gg 1$, whereas if $\lambda$ is small, at different locations $Y$ the waves may effectively be in a low viscosity $\hat{\lambda} \ll 1$ or a viscous $\hat{\lambda} \gg 1$ regime. In a viscous regime we have

$$
\nu_{\text {eff }}=-\frac{1}{2} m^{-6} \lambda^{-2} \quad(\hat{\lambda} \gg 1) .
$$

This destabilising negative viscosity term corresponds to the instability of the original steady Kolmogorov flow discussed in Meshalkin \& Sinai (1961), and follows from the anisotropy of the forcing as discussed for example in Srinivasan \& Young (2014). The inverse power of $\lambda$ shows the effect of large viscosity in cutting off the feedback on the mean flow, corresponding to capturing the short-time behaviour seen in figure $1(\mathrm{~d})$. This result is key to zonostrophic instability, in which $\Omega \simeq 0$ initially everywhere. No matter what the molecular value of $\lambda>0$, the limit $\hat{\lambda} \gg 1$ and the result (3.30) are then appropriate, with the destabilising effect of a negative effective viscosity arising from the negative values of $f_{\mathrm{R}}$ for short times and so of $h_{\mathrm{R}}$ for large $\hat{\lambda}$; see figure 2 . 


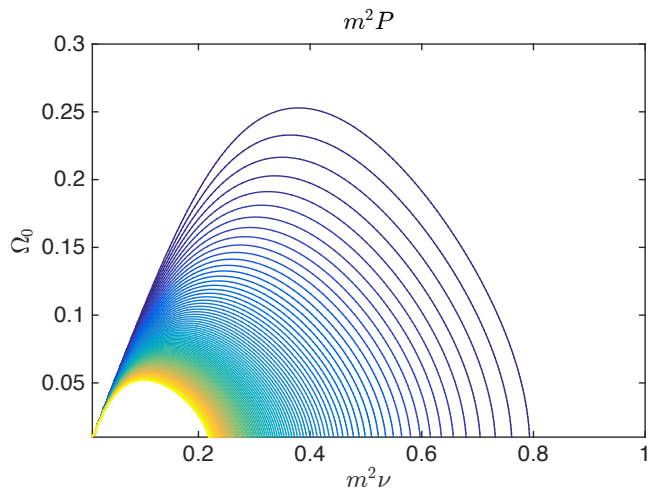

Figure 3. Contour plot of $m^{2} P(3.32)$ in the $\left(m^{2} \lambda, \Omega_{0}\right)$-plane. Contours increase from $m^{2} P=0$ (outermost) in steps of 0.1 to $m^{2} P=10$ (bottom left corner).

We remark that even if we applied molecular hyperviscosity (i.e., hyperdiffusion) to the waves (end of section 2.3), which would lead to modified forms of $D$ and $\mathcal{L}$, we would still recover effective viscosity for the large scale fields, in other words via a term of the form $\partial_{Y}\left(\nu_{\text {eff }} \partial_{Y} \Omega\right)$. Whereas negative effective viscosity leads to an ill-posed problem if it is only balanced by weaker positive molecular viscosity, having molecular hyperviscosity recovers a sensible problem for the large scale fields, for example to integrate numerically. We leave this for future study, noting that molecular hyperviscosity should only be introduced with considerable caution: see Sukoriansky, Galperin \& Chekhlov (1999) in the case of turbulence and Zhang \& Jones (1997) for rapidly rotating convection.

\subsection{Zonostrophic instabilty}

At the end of the last section we indicated the origin of zonostrophic instability through the negative sign in (3.30). We study this in the more general context of a uniform background shear flow. Consider again equation (3.28) and think of $\nu_{\text {eff }}=\nu_{\text {eff }}(\Omega, \lambda)$ in general. A solution is simply steady uniform vorticity $\Omega(Y, \mathcal{T})=\Omega_{0}=$ const., that is uniform shear $U=-\Omega_{0} Y$, and we can investigate the stability of this solution to perturbations.

We replace $\Omega \rightarrow \Omega_{0}+\Omega$ in (3.28) with now $\Omega \ll 1$ to give

$$
\partial_{\mathcal{T}} \Omega=\partial_{Y}\left[\nu_{\mathrm{eff}} \partial_{Y} \Omega\right]+\lambda \partial_{Y}^{2} \Omega, \quad \nu_{\mathrm{eff}}=\nu_{\mathrm{eff}}\left(\Omega_{0}, \lambda\right)
$$

at leading order. Seeking a normal mode in $\Omega$ proportional to $\exp \left(P K^{2} \mathcal{T}+i K Y\right)$ gives the (scaled) growth rate $P$ as

$$
P=-\nu_{\text {eff }}\left(\Omega_{0}, \lambda\right)-\lambda=-m^{-2} \Omega_{0}^{-2} h_{\mathrm{R}}\left(m^{2} \lambda / \Omega_{0}\right)-\lambda .
$$

The last term $-\lambda$ is the damping effect of molecular viscosity and the nature of any instability is again clear as the result of a negative effective viscosity, taking place if $\nu_{\text {eff }}$ is negative and large enough. First consider when $\Omega_{0}=0$, in which case we insert the large $\hat{\lambda}$ expansion (3.30) to obtain

$$
P=\frac{1}{2} m^{-6} \lambda^{-2}-\lambda .
$$

We have zonostrophic instability if $P>0$ which amounts to the condition $m^{2} \lambda<2^{-1 / 3}$.

For the more general case of a uniform background vorticity distribution $\Omega_{0} \neq 0$, the relative size of $\Omega_{0}$ and $\lambda$ is important through the value $\hat{\lambda}=m^{2} \lambda / \Omega_{0}$ in (3.32). We cannot evaluate $h_{\mathrm{R}}$ analytically in general, but we can easily do so numerically, and figure 
14

(a)

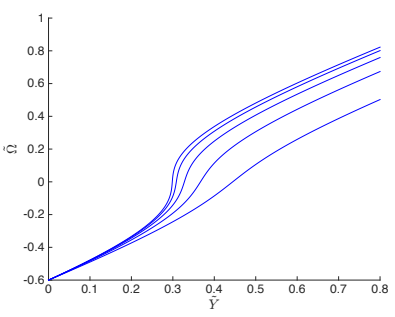

(b)

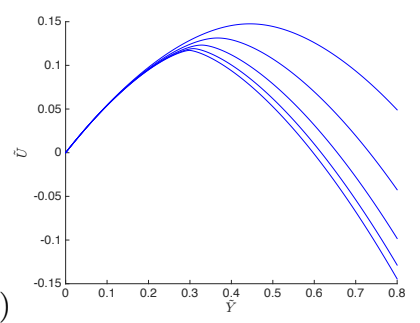

(c)

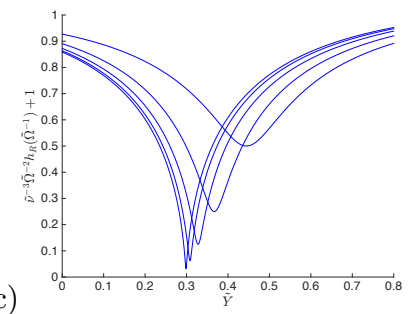

Figure 4. Plots of equilibrium profiles found by integrating the ODEs (3.36). Here $2-\tilde{\lambda}^{-3}=1$, $\frac{1}{2}, \frac{1}{4}, \ldots \frac{1}{16}$, and as $\tilde{\lambda}^{-3}$ approaches the value 2 , smaller scale structure is evident in the curves for (a) $\tilde{\Omega}(\tilde{Y})$ (reading up the curves), (b) $\tilde{U}(\tilde{Y})$ (down), and (c) $\tilde{\lambda}^{-3} \tilde{\Omega}^{-2} h_{\mathrm{R}}\left(\tilde{\Omega}^{-1}\right)+1$ (down, on the left-hand side)

2 shows a contour plot of $m^{2} P$ in the $\left(m^{2} \lambda, \Omega_{0}\right)$-plane. The outer contour gives $P=0$ and outside this $P<0$. Along the horizontal axis $\Omega_{0}=0$ the result (3.33) holds. For increasing $\Omega_{0}$, zonostrophic instability is suppressed (Srinivasan \& Young 2014; Hsu \& Diamond 2015), being restricted to a finite range of viscosities $m^{2} \lambda$ bounded above zero, and turns off completely for $\Omega_{0}$ greater than about 0.25 . Note that the suppression of instability by shear is also evident in the sign of the second, correction term in (3.29). The value of $m^{2} P$ diverges as $\lambda \rightarrow 0, \Omega_{0} \rightarrow 0$ (bottom left, where the contours accumulate), and in fact they accumulate near the origin on the line given by $m^{2} \lambda / \Omega_{0} \simeq 0.95$, on which $h_{\mathrm{R}}$ changes sign.

\subsection{Equilibrium profiles}

We cannot readily time step (3.28) numerically: as $\nu_{\text {eff }}$ can change sign this is ill-posed without some additional cut-off for short wavelengths (on the $Y$ scale). Incorporating a cut-off would not be unreasonable given that the theory is based on scale separation and a threshold can be determined in related models which allow zonostrophic instability on arbitrary scales (Srinivasan \& Young 2012). For example negative effective viscosity at large scale can be cut off by positive effective hyperviscosity at smaller scales (and Sukoriansky, Galperin \& Chekhlov (1999) argue that in two-dimensional turbulence these effects also need to be time-dependent to simulate subgrid-scale motion). We defer these considerations to future study, and here note that although we cannot integrate (3.28) explicitly, we can seek equilibrium profiles, that is, mean field profiles independent of the time scale $\mathcal{T}$. Integrating (3.28) once, we can write

$$
m^{2}\left(\lambda+\nu_{\mathrm{eff}}\right) \partial_{Y} \Omega \equiv\left[m^{2} \lambda+\Omega^{-2} h_{\mathrm{R}}\left(m^{2} \lambda / \Omega\right)\right] \partial_{Y} \Omega=C,
$$

with a constant $C$. To plot solutions it is convenient to reduce the number of parameters by setting

$$
\Omega=m^{2} \lambda \tilde{\Omega}, \quad U=m^{6} \lambda^{3} C^{-1} \tilde{U}, \quad Y=m^{4} \lambda^{2} C^{-1} \tilde{Y}, \quad \lambda=m^{-2} \tilde{\lambda}
$$

so that we solve

$$
\left[1+\tilde{\lambda}^{-3} \tilde{\Omega}^{-2} h_{\mathrm{R}}\left(\tilde{\Omega}^{-1}\right)\right] \partial_{\tilde{Y}} \tilde{\Omega}=1, \quad \partial_{\tilde{Y}} \tilde{U}=-\tilde{\Omega},
$$

to obtain a family of solutions depending on only one parameter $\tilde{\lambda}$.

Now we numerically integrate (3.36) over a range of $\tilde{Y}$ with $\tilde{\Omega}$ passing through $\tilde{\Omega}=0$ : this gives a family of profiles depicted in figure $4($ a) for $\tilde{\Omega}$ and (b) for $\tilde{U}$. It turns out that we cannot obtain sensible solutions unless the effective viscosity $[\cdots]$ in (3.36) remains positive, and this restricts $\tilde{\lambda}^{-3}<2$. This is equivalent to being below the threshold for zonostrophic instability (without background uniform shear) in (3.33). As $\tilde{\lambda}^{-3}$ is made 
closer to 2 we have a lower effective viscosity in a region close to $\tilde{\Omega}=0$ and so the scope to sustain larger gradients of $\tilde{\Omega}$. This is seen in panel (a) of figure 4 , while panel (b) shows the increasing sharpness of the $\tilde{U}$ profile and panel (c) shows the corresponding dip in the total viscosity, i.e. $\lambda+\nu_{\text {eff }}$, to a small but positive value.

Using (3.29) we can also obtain the approximate form of the $\tilde{\Omega}$ profile as an inverse cubic,

$$
\frac{5}{2} \tilde{\lambda}^{-3} \tilde{\Omega}^{3}+\left(1-\frac{1}{2} \tilde{\lambda}^{-3}\right) \tilde{\Omega}=\tilde{Y}
$$

and in the limit $\hat{\lambda}^{-3} \rightarrow 2$ we obtain profiles

$$
\tilde{\Omega}=(\tilde{Y} / 5)^{1 / 3}, \quad \tilde{U}=-\frac{3}{4} 5^{-1 / 3}|\tilde{Y}|^{4 / 3}
$$

(up to appropriate additive constants).

\section{Passive scalar and kinematic magnetic field transport}

As a useful intermediate step before tackling the full MHD problem, but also of interest in its own right, we consider passive scalar evolution; related work including bounds on effective diffusivities may be found in Srinivasan \& Young (2014), when the vorticity is damped by a bottom drag term $-\lambda_{0} \omega$ and likewise the passive scalar field, i.e. via a term $-\lambda_{0} \sigma$. Naturally, for us the passive scalar could be the vector potential of a weak magnetic field, in a kinematic regime; see $(2.2,2.3)$ and below.

\subsection{Multiple scale formulation}

We follow the discussion at the beginning of section 3 and set $\sigma(y, t)=e^{-i m U(y) t} c(y, t)$. With the scalings

$$
\begin{aligned}
& \Sigma(y) \rightarrow \Sigma(Y), \quad \sigma \rightarrow \varepsilon^{3 / 2} \sigma(Y, T), \quad c \rightarrow \varepsilon^{3 / 2} c(Y, T), \\
& F_{\mathrm{P}} \rightarrow \varepsilon^{3} F_{\mathrm{P}}(Y, T) \quad \mathcal{F}_{\mathrm{P}} \rightarrow \varepsilon^{2} \mathcal{F}_{\mathrm{P}}(Y), \quad \lambda \rightarrow \varepsilon \lambda,
\end{aligned}
$$

(and $Y, T$ as before) we obtain from (2.13),

$$
\partial_{T} c=i m \Sigma^{\prime} \phi+\lambda \mathcal{L} c
$$

for the waves and

$$
\partial_{\mathcal{T}} \Sigma+\partial_{Y} \mathcal{F}_{\mathrm{P}}=\lambda \partial_{Y}^{2} \Sigma
$$

for the mean field, from (2.22). The scalings for the fluxes in (4.2) differ from those earlier in (3.8) because

$$
F_{\mathrm{P}}=i m\left(\phi^{*} c-\phi c^{*}\right)
$$

does not have the same leading order cancellation as that seen for $F_{\mathrm{R}}$, discussed below (3.5). Although the equations for vorticity and a passive scalar have similarities this is one key difference, the other being that the vorticity equation includes an explicit source term, the external forcing, where for a passive scalar there is no source, only a background gradient.

The leading order passive scalar problem is easily integrated, using (3.23),

$$
\begin{aligned}
c_{0} & =\Sigma^{\prime} m^{-1} \Omega^{-1} \hat{\beta}^{-1} D\left[\exp \left(i \hat{\beta} \tan ^{-1} \tau\right)-1\right], \\
F_{\mathrm{P}} & =-m^{-2} \Sigma^{\prime} \Omega^{-1} D^{2} f_{\mathrm{P}}, \quad f_{\mathrm{P}}=2 \hat{\beta}^{-1}\left(1+\tau^{2}\right)^{-1} \sin \left(\hat{\beta} \tan ^{-1} \tau\right),
\end{aligned}
$$

(with $D$ defined in (3.22)) and we may write the integrated flux as

$$
\mathcal{F}_{\mathrm{P}}=-\kappa_{\mathrm{eff}} \Sigma^{\prime}, \quad \kappa_{\mathrm{eff}}=m^{-2} \Omega^{-2} h_{\mathrm{P}},
$$


(a)

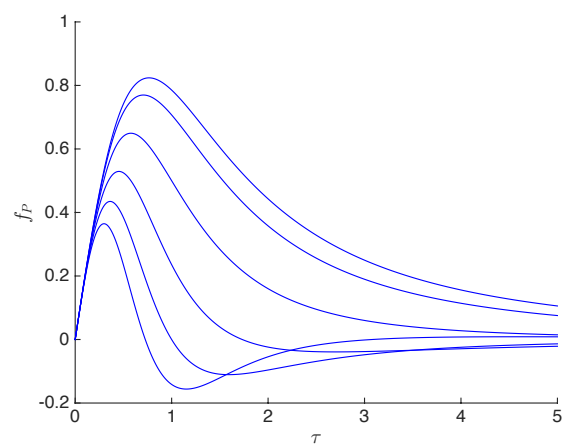

(b)

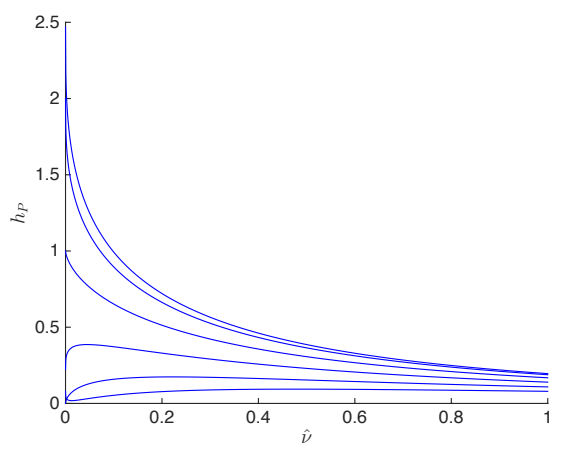

Figure 5. Plot of (a) $f_{\mathrm{P}}$ as a function of $\tau$ and (b) of $h_{\mathrm{P}}$ as a function of $\hat{\lambda}$. In each plot values are $\hat{\beta}=0,1, \ldots 5$, reading down the curves on the left.

(a)

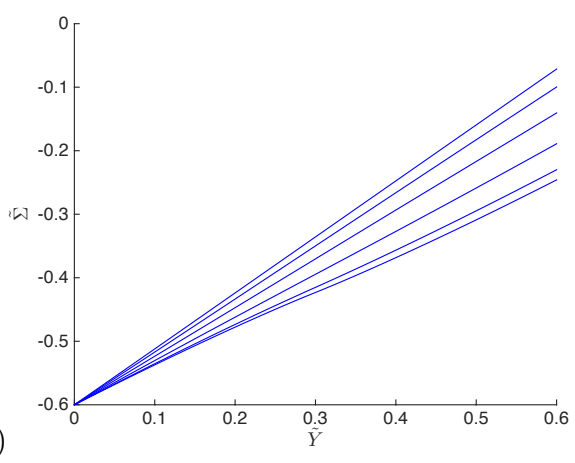

(b)

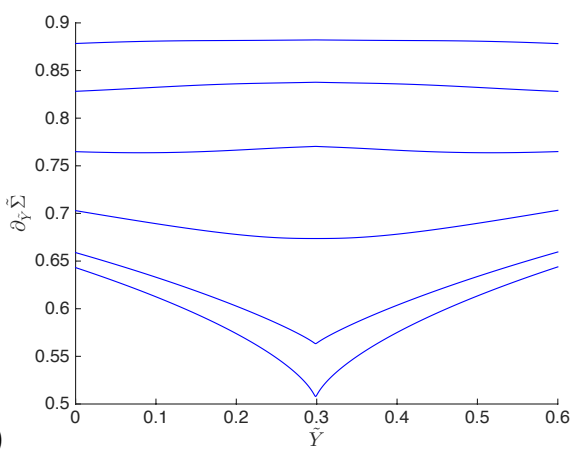

Figure 6 . Plots of equilibrium scalar profiles by integrating the ODE (4.13). Here $2-\tilde{\lambda}^{-3}=\frac{1}{16}$. For values of $\tilde{\beta}=0,1, \ldots, 5$, in (a) $\tilde{\Sigma}(\tilde{Y})$ (reading up the curves) and (b) $\partial_{\tilde{Y}} \tilde{\Sigma}$ (reading up) are plotted against $\tilde{Y}$.

with $h_{\mathrm{P}}=h_{\mathrm{P}}(\hat{\lambda}, \hat{\beta})$ from (3.27). This appears in the large scale passive scalar transport equation

$$
\partial_{\mathcal{T}} \Sigma=\partial_{Y}\left[\kappa_{\mathrm{eff}} \partial_{Y} \Sigma\right]+\lambda \partial_{Y}^{2} \Sigma
$$

As in the previous section, with similar notation, the function $f_{\mathrm{P}}$ gives the instantaneous flux of passive scalar (from a uniform background) in the presence of a wave initiated by the external forcing at $T=0$. Unlike in the previous setion $f_{\mathrm{P}}$ depends on the parameter $\hat{\beta}$ as well as rescaled time $\tau$, but not on $\hat{\lambda}$. Figure 5 (a) shows $f_{P}$ for varying values of $\hat{\beta}$ : as this parameter is increased the profile becomes increasingly oscillatory and the integrated transport is reduced. This is shown in figure 5(b) depicting $h_{\mathrm{P}}$ as a function of $\hat{\lambda}$ for various values of $\hat{\beta}$, showing the suppression of transport as the $\beta$ effect is increased (see, e.g. Srinivasan \& Young 2014) and the flows become more wave-like in nature; we also note that

$$
h_{\mathrm{P}}(0, \hat{\beta})=2 \hat{\beta}^{-2}[1-\cos (\pi \hat{\beta} / 2)], \quad h_{\mathrm{P}}(\hat{\lambda}, \hat{\beta})=\frac{1}{2} \hat{\lambda}^{-2}-\left(2+\frac{1}{8} \hat{\beta}^{2}\right) \hat{\lambda}^{-4}+\cdots \quad(\hat{\lambda} \gg 1) .
$$

\subsection{Equilibrium profiles}

Although in figure 5 we see variation in transport of passive scalar as $\hat{\lambda}$ and $\hat{\beta}$ are varied, the effect on equilibrium passive scalar profiles turns out not to be as dramatic 
as for vorticity transport in section 3.5. The steady state version of (4.9) is

$$
m^{2}\left[\lambda+\kappa_{\mathrm{eff}}\right] \partial_{Y} \Sigma=\left[m^{2} \lambda+\Omega^{-2} h_{\mathrm{P}}\left(m^{2} \lambda / \Omega, \beta / m \Omega\right)\right] \partial_{Y} \Sigma=C_{\Sigma}
$$

where the constant $C_{\Sigma}$ would be fixed by the overall gradient of passive scalar across the system. We rescale

$$
\Sigma=m^{2} \lambda C_{\Sigma} C^{-1} \tilde{\Sigma}, \quad \beta=m^{3} \lambda \tilde{\beta},
$$

together with (3.35), so that we can write from (4.11),

$$
\left[1+\tilde{\lambda}^{-3} \tilde{\Omega}^{-2} h_{\mathrm{P}}\left(\tilde{\Omega}^{-1}, \tilde{\beta} \tilde{\Omega}^{-1}\right)\right] \partial_{\tilde{Y}} \tilde{\Sigma}=1
$$

To obtain a passive scalar profile we first fix $\tilde{\lambda}^{-3}=2-\frac{1}{16}$ giving the sharpest $\tilde{\Omega}$ profile depicted in figure 4(a). We then integrate (4.13) for any value of $\tilde{\beta}$ and the resulting $\tilde{\Sigma}(\tilde{Y})$ are shown in figure $6(\mathrm{a})$ for $\tilde{\beta}=0,1, \ldots 5$, reading up the curves. There is little effect on sharpening the passive scalar profiles in panel (a), although plots of the gradient $\partial_{\tilde{Y}} \tilde{\Sigma}$ in panel (b) show more structure. Note that if the passive scalar is the vector potential of a kinematic magnetic field, then figure 5(b) represents the field itself, reduced to approximately half the exterior value within the step for $\beta=0$. This may be thought of as a process of flux expulsion (Weiss 1966), from regions of stronger motion corresponding to greater effective scalar diffusivity.

Somewhat paradoxically then, a step jump can be supported in the vorticity profile of figure 4(a), whereas a passive scalar profile remains relatively unperturbed. Of course different types of transport are involved, and whereas the effective viscosity can become negative and nearly cancel out the molecular viscosity to allow a jump, the same is not true for the passive scalar (or vector potential). Further investigations for the case of different values of scalar diffusivity and viscosity in (2.7) would be of interest, but the governing ODEs would need to be solved numerically.

\section{MHD transport}

We now return to the full MHD problem given in section 2.2, armed with the above discussion of the hydrodynamic and passive scalar/kinematic magnetic field cases. The scalings remain the same for the vorticity as in section 3 and for the vector potential the development follows that for the passive scalar in section 4, though naturally we now have the Lorentz force coupling. We will drop further mention of passive scalar from the discussion.

\subsection{Multiple scale formulation}

We again remove the fast advection terms in $(2.11,2.12)$, by setting

$$
(\omega, \psi, j, a)(y, t)=e^{-i m U(y) t}(\zeta, \phi, \gamma, \alpha)(y, t),
$$

(as listed in table 1) to obtain

$$
\begin{aligned}
& \partial_{t} \zeta-i m B \gamma=i m\left(\beta+\Omega^{\prime}\right) \phi-i m J^{\prime} \alpha+\lambda \mathcal{L} \zeta, \\
& \partial_{t} \alpha-i m B \phi=\lambda \mathcal{L} \alpha, \\
& \zeta=-\mathcal{L} \phi, \quad \gamma=-\mathcal{L} \alpha,
\end{aligned}
$$

with corresponding fluxes $F_{\mathrm{R}}$ in (3.5),

$$
\begin{aligned}
F_{\mathrm{L}} & =\partial_{y}\left[2 m^{2} \Omega t|\alpha|^{2}-i m\left(\alpha^{*} \partial_{y} \alpha-\alpha \partial_{y} \alpha^{*}\right)\right], \\
F_{\mathrm{M}} & =i m\left(\phi^{*} \alpha-\phi \alpha^{*}\right) .
\end{aligned}
$$


For the large scale expansion we replace

$$
U(y) \rightarrow U(Y), \quad \Omega(y) \rightarrow \varepsilon \Omega(Y), \quad A(y) \rightarrow A(Y), \quad B(y) \rightarrow \varepsilon B(Y) .
$$

As before we adopt $Y=\varepsilon y, T=\varepsilon t, \mathcal{T}=\varepsilon^{3} t$, as well as the replacements

$$
\lambda \rightarrow \varepsilon \lambda, \quad \beta \rightarrow \varepsilon \beta, \quad(\zeta, \phi, \gamma, \alpha) \rightarrow \varepsilon^{3 / 2}(\zeta, \phi, \gamma, \alpha)(Y, T),
$$

referring to the small scale fields and

$$
\left(F_{\mathrm{R}}, F_{\mathrm{L}}, F_{\mathrm{M}}\right) \rightarrow\left(\varepsilon^{4} F_{\mathrm{R}}, \varepsilon^{4} F_{\mathrm{L}}, \varepsilon^{3} F_{\mathrm{M}}\right)(Y, T) \quad\left(\mathcal{F}_{\mathrm{R}}, \mathcal{F}_{\mathrm{L}}, \mathcal{F}_{\mathrm{M}}\right) \rightarrow\left(\varepsilon^{3} \mathcal{F}_{\mathrm{R}}, \varepsilon^{3} \mathcal{F}_{\mathrm{L}}, \varepsilon^{2} \mathcal{F}_{\mathrm{M}}\right)(Y, T)
$$

for the fluxes. This yields, exactly

$$
\begin{aligned}
\partial_{T} \zeta-i m B \gamma & =i m\left(\beta+\varepsilon \Omega^{\prime}\right) \phi-i m \varepsilon^{2} J^{\prime} \alpha+\lambda \mathcal{L} \zeta, \\
\partial_{T} \alpha-i m B \phi & =\lambda \mathcal{L} \alpha,
\end{aligned}
$$

with the large scale equations

$$
\begin{aligned}
& \partial_{\mathcal{T}} \Omega+\partial_{Y} \mathcal{F}_{\mathrm{K}}=\lambda \partial_{Y}^{2} \Omega, \\
& \partial_{\mathcal{T}} A+\partial_{Y} \mathcal{F}_{\mathrm{M}}=\lambda \partial_{Y}^{2} A,
\end{aligned}
$$

recalling (2.23). The flux $F_{\mathrm{R}}$ is given in (3.11), we gain a similar term from the Lorentz force,

$$
F_{\mathrm{L}}=\partial_{Y}\left[2 m^{2} \Omega T|\alpha|^{2}-i \varepsilon m\left(\alpha^{*} \partial_{Y} \alpha-\alpha \partial_{Y} \alpha^{*}\right)\right],
$$

and $F_{\mathrm{M}}$ remains as in (5.6).

\subsection{Numerical solution}

Again, before embarking on analysis, we present a simulation of the full wave problem with $\beta=0, \varepsilon=0.1, U=\cos Y$ and a constant field $B=2$. The exact, rescaled equations are

$$
\begin{aligned}
& \partial_{T} \omega+i m \varepsilon^{-1} U \omega=i m B j+i m\left(\beta+\varepsilon \Omega^{\prime}\right) \psi-i m \varepsilon^{2} J^{\prime} a+\lambda\left(\varepsilon^{2} \partial_{Y}^{2}-m^{2}\right) \omega \\
& \partial_{T} a+i m \varepsilon^{-1} U a=i m B \psi+\lambda\left(\varepsilon^{2} \partial_{Y}^{2}-m^{2}\right) a \\
& -\omega=\left(\varepsilon^{2} \partial_{Y}^{2}-m^{2}\right) \psi, \quad-j=\left(\varepsilon^{2} \partial_{Y}^{2}-m^{2}\right) a .
\end{aligned}
$$

(The fluxes $F_{\mathrm{R}}, F_{\mathrm{L}}$ are rescaled as in (3.17) whereas $F_{\mathrm{P}}$ is not.) Figure 7 shows the real parts of the fields $\omega, \psi, j, a$ as functions of $(T, Y)$, in panels (a-d) with the corresponding fluxes in panels $(\mathrm{e}-\mathrm{h})$. As well as the general decrease in scale as $T$ increases, one can clearly see the presence of oscillations, Alfvén waves, in which energy is transferred between fluid flow and magnetic field in $(\mathrm{a}-\mathrm{d})$. With these are oscillations in the instantaneous fluxes giving the feedback on the mean flow and field.

\subsection{Multiple scale expansion}

To analyse the complicated picture emerging in figure 7 , we again expand all small scale fields in powers of $\varepsilon$ to yield the leading order wave equations, from $(5.10,5.11)$,

$$
\begin{aligned}
& \partial_{T} \zeta_{0}-i m B \gamma_{0}=i m \beta \phi_{0}-\lambda m^{2}\left(1+\Omega^{2} T^{2}\right) \zeta_{0}, \\
& \partial_{T} \alpha_{0}-i m B \phi_{0}=-\lambda m^{2}\left(1+\Omega^{2} T^{2}\right) \alpha_{0}, \\
& \zeta_{0}=m^{2}\left(1+\Omega^{2} T^{2}\right) \phi_{0}, \quad \gamma_{0}=m^{2}\left(1+\Omega^{2} T^{2}\right) \alpha_{0} .
\end{aligned}
$$




\section{Page 19 of 35}

(a)

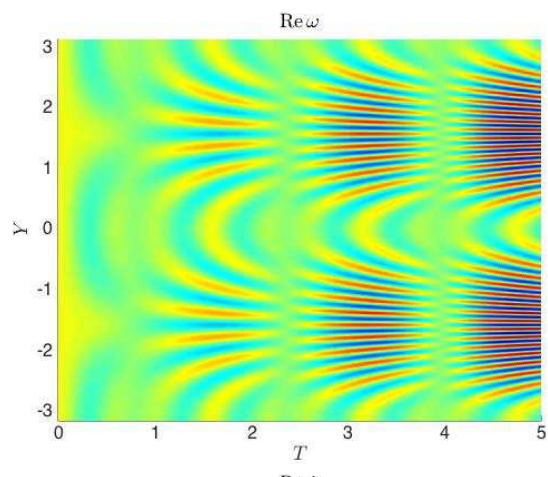

(c)

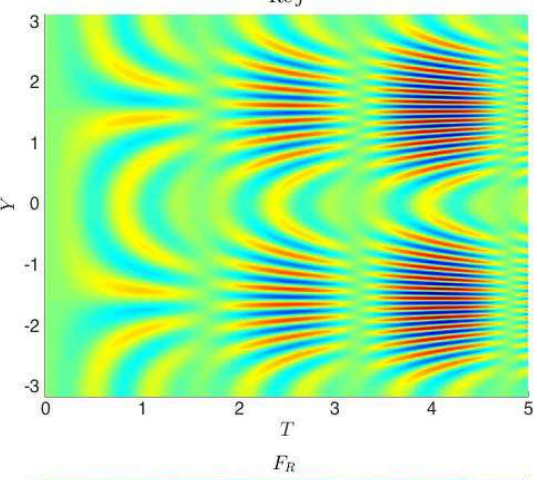

(e)
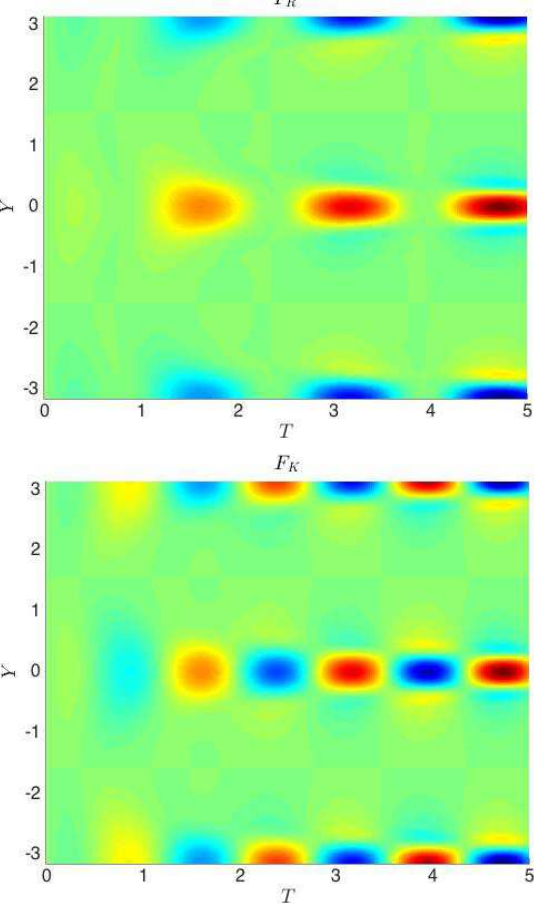

(b)

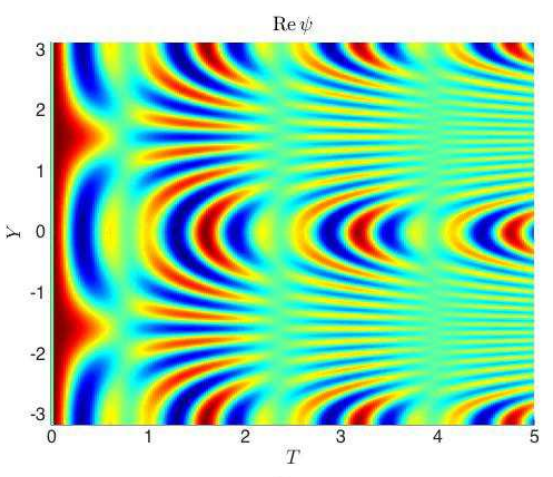

(d)

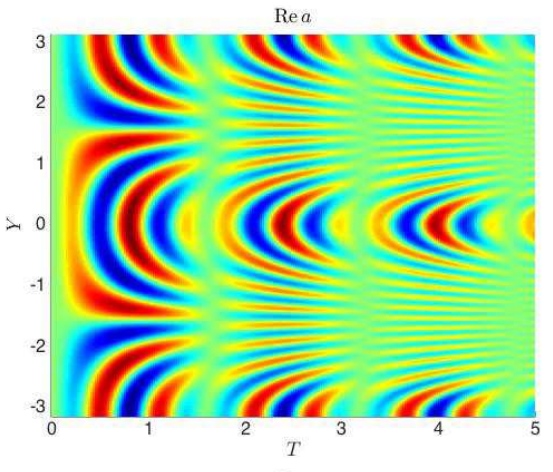

$F_{L}$

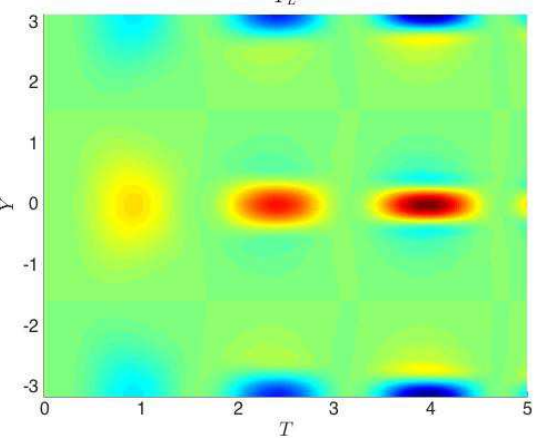

$F_{M}$

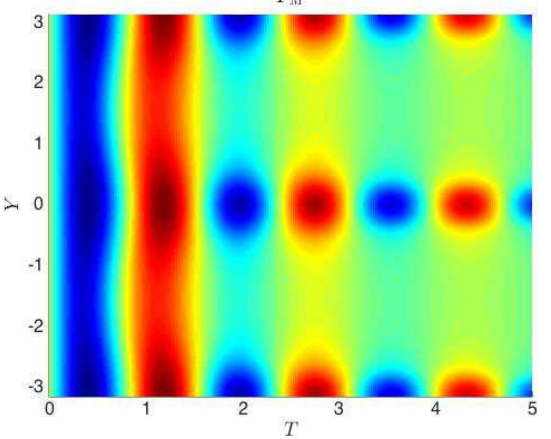

(h)

(g)

Figure 7. Simulation of waves with $\beta=0, m=1, \varepsilon=0.1, \lambda=0, U=\cos Y$ and $B=2$. In (a) $\operatorname{Re} \omega,(\mathrm{b}) \operatorname{Re} \psi,(\mathrm{c}) \operatorname{Re} j$, (d) $\operatorname{Re} a,(\mathrm{e}) F_{\mathrm{R}},(\mathrm{f}) F_{\mathrm{L}}$, (g) $F_{\mathrm{K}}$, and (h) $F_{\mathrm{M}}$ are plotted in the ( $T, Y)$-plane. 
20

These are to be solved with initial conditions from (2.15) and the results fed into the mean equations via fluxes (dropping the terms of order $\varepsilon$ ),

$$
F_{\mathrm{R}}=2 m^{2} \partial_{Y}\left(\Omega T\left|\phi_{0}\right|^{2}\right), \quad F_{\mathrm{L}}=2 m^{2} \partial_{Y}\left(\Omega T\left|\alpha_{0}\right|^{2}\right), \quad F_{\mathrm{M}}=i m\left(\phi_{0}^{*} \alpha_{0}-\phi_{0} \alpha_{0}^{*}\right)
$$

Unfortunately the above ODEs (5.18-5.20) are not soluble analytically in simple terms (that is, without recourse to so-called Heun functions). They can be solved approximately in the limit of strong and weak shear as measured by $\hat{B}$ below (Leprovost \& Kim 2009) to obtain expressions for the effective transport coefficients for the case of isotropic driving.

In the first instance we wish to proceed without approximation. We first rescale and remove the diffusive decay by setting

$$
\left(\zeta_{0}, \phi_{0}, \gamma_{0}, \alpha_{0}\right)=\left(\hat{\zeta}_{0}, m^{-2} \hat{\phi}_{0}, \hat{\gamma}_{0}, m^{-2} \hat{\alpha}_{0}\right) D
$$

with the damping term $D$ given in (3.22), to leave equations for sheared Alfvén waves,

$\partial_{T} \hat{\zeta}_{0}-i m B \hat{\gamma}_{0}=i m^{-1} \beta \hat{\phi}_{0}, \quad \partial_{T} \hat{\alpha}_{0}-i m B \hat{\phi}_{0}=0, \quad \hat{\zeta}_{0}=\left(1+\Omega^{2} T^{2}\right) \hat{\phi}_{0}, \quad \hat{\gamma}_{0}=\left(1+\Omega^{2} T^{2}\right) \hat{\alpha}_{0}$.

Although we cannot solve the ODEs, we proceed for the moment as if we knew the solution with all quantities expressed as functions of $(T, \Omega, B, \beta)$. We would then evaluate the fluxes by differentiating these expressions, and could write the instantaneous fluxes in the form

$$
\begin{aligned}
& F_{\mathrm{R}}=-m^{-2} \Omega^{\prime} \Omega^{-1} D^{2} f_{\Omega \mathrm{R}}-m^{-2} B^{\prime} B^{-1} D^{2} f_{B \mathrm{R}} \\
& F_{\mathrm{L}}=-m^{-2} \Omega^{\prime} \Omega^{-1} D^{2} f_{\Omega \mathrm{L}}-m^{-2} B^{\prime} B^{-1} D^{2} f_{B \mathrm{~L}} \\
& F_{\mathrm{M}}=-m^{-2} B \Omega^{-1} D^{2} f_{\mathrm{M}}
\end{aligned}
$$

Note that crucially, the fluxes depend on $Y$ not only through gradients of the vorticity profile $\Omega^{\prime}$ (as in the hydrodynamic problem earlier) but also through gradients $B^{\prime}$ in the magnetic field profile. This feature, discussed by Chechkin (1999), Kim (2007) and Leprovost \& Kim (2009), creates additional complexity in the MHD problem

Following through with similar notation to section 3.3, the functions $f_{\mathrm{Z}}$ encapsulate all the interesting time evolution of the fluxes, and this may be most conveniently expressed by first extending the definitions in (3.22) to include

$$
\hat{B}=m B / \Omega \text {. }
$$

We may then write all the functions in terms of $\tau$ and three parameters $\hat{B}, \hat{\beta}$ and $\hat{\lambda}$, with

$$
\begin{aligned}
f_{\Omega \mathrm{R}} & =-2 \tau\left(1+\tau^{2}\right)^{-2}\left[\left(1-3 \tau^{2}\right)\left(1+\tau^{2}\right)^{-1}-\frac{4}{3} \hat{\lambda} \tau^{3}+\Omega \partial_{\Omega}\right]\left|\hat{\zeta}_{0}\right|^{2} \\
f_{\Omega \mathrm{L}} & =-2 \tau\left[1-\frac{4}{3} \hat{\lambda} \tau^{3}+\Omega \partial_{\Omega}\right]\left|\hat{\alpha}_{0}\right|^{2} \\
f_{B \mathrm{R}} & =-2 \tau\left(1+\tau^{2}\right)^{-2} B \partial_{B}\left|\hat{\zeta}_{0}\right|^{2} \\
f_{B \mathrm{~L}} & =-2 \tau B \partial_{B}\left|\hat{\alpha}_{0}\right|^{2} \\
f_{\mathrm{M}} & =-\left(1+\tau^{2}\right)^{-1} \hat{B}^{-1} i\left(\hat{\zeta}_{0}^{*} \hat{\alpha}_{0}-\hat{\zeta}_{0} \hat{\alpha}_{0}^{*}\right) .
\end{aligned}
$$

Here the functions $f_{\Omega \mathrm{R}}$ and $f_{\Omega \mathrm{L}}$ capture the effect of a vorticity gradient $\Omega^{\prime}$ on the Reynolds stress and Lorentz force, while $f_{B \mathrm{R}}$ and $f_{B \mathrm{~L}}$ give the effect in the NavierStokes equation of a gradient of background magnetic field $B^{\prime}$. The transport of scalar vector potential $A$ is linked to $B=A^{\prime}$ through the $f_{\mathrm{M}}$ term.

We now extend the convention in $(2.23)$, so that $\mathrm{Z}_{\mathrm{K}}=\mathrm{Z}_{\mathrm{R}}-\mathrm{Z}_{\mathrm{L}}$ for any quantity $\mathrm{Z}$, and express the integrated fluxes in terms of transport coefficients $\nu_{\text {eff }}, \chi_{\text {eff }}$ and $\eta_{\text {eff }}$,

$$
\mathcal{F}_{\mathrm{K}}=-\nu_{\mathrm{eff}} \Omega^{\prime}-\chi_{\mathrm{eff}} B^{\prime}, \quad \mathcal{F}_{\mathrm{M}}=-\eta_{\mathrm{eff}} B
$$


(a)

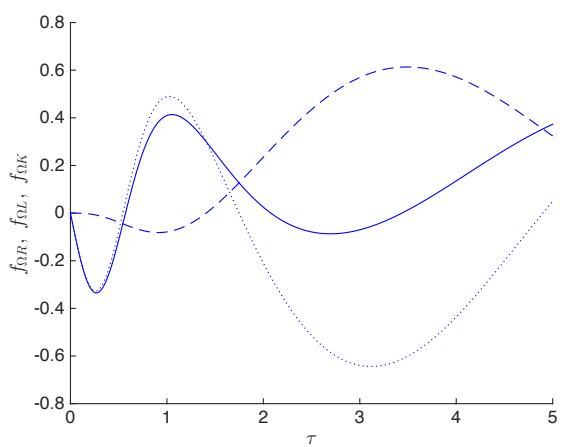

(b)

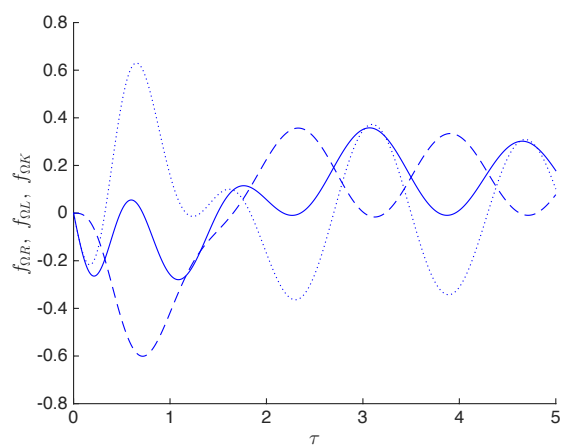

Figure 8. Plots of $f_{\Omega \mathrm{R}}$ (solid), $f_{\Omega \mathrm{L}}$ (dash) and $f_{\Omega \mathrm{K}}$ (dot) as functions of $\tau$ for $\hat{\lambda}=\hat{\beta}=0$ and (a) $\hat{B}=0.5$ and (b) $\hat{B}=2.0$.

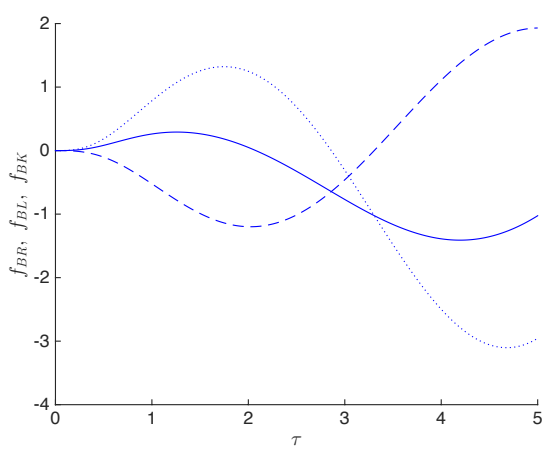

(a) (b)

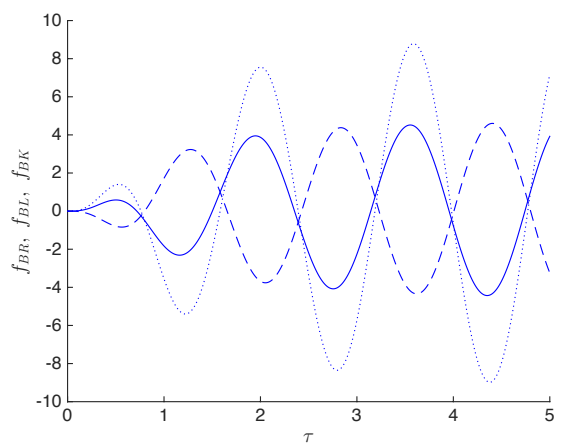

Figure 9. Plots of $f_{B R}$ (solid), $f_{B \mathrm{~L}}$ (dash) and $f_{B \mathrm{~K}}$ (dot) as functions of $\tau$ for $\hat{\lambda}=\hat{\beta}=0$ and (a) $\hat{B}=0.5$ and (b) $\hat{B}=2.0$.

This leads to coupled large scale equations

$$
\begin{aligned}
& \partial_{\mathcal{T}} \Omega=\partial_{Y}\left[\nu_{\mathrm{eff}} \partial_{Y} \Omega+\chi_{\mathrm{eff}} \partial_{Y} B\right]+\lambda \partial_{Y}^{2} \Omega, \\
& \partial_{\mathcal{T}} A=\partial_{Y}\left[\eta_{\mathrm{eff}} \partial_{Y} A\right]+\lambda \partial_{Y}^{2} A,
\end{aligned}
$$

with (making use of (3.27)),

$$
\nu_{\text {eff }}=m^{-2} \Omega^{-2} h_{\Omega \mathrm{K}}, \quad \chi_{\mathrm{eff}}=m^{-2} \Omega^{-1} B^{-1} h_{B \mathrm{~K}}, \quad \eta_{\mathrm{eff}}=m^{-2} \Omega^{-2} h_{\mathrm{M}} .
$$

We have reached the end of an analytical development for the MHD problem mirroring that for the hydrodynamic framework in section 3.3. If we could solve the ODEs (5.23) explicitly to obtain $\hat{\zeta}_{0}, \hat{\phi}_{0}, \hat{\alpha}_{0}, \hat{\gamma}_{0}$, taken as functions of $(T, \Omega, B, \beta)$, we would then calculate the functions $f_{\mathrm{Z}}$ in (5.28-5.32), differentiating quantities as needed. Although we cannot solve these ODEs analytically in general, we can do so numerically, and in fact we can add to these ODEs further equations for derivatives such as $\Omega \partial_{\Omega}\left|\hat{\zeta}_{0}\right|^{2}$ or $B \partial_{B}\left|\hat{\zeta}_{0}\right|^{2}$. We relegate these uninspiring details to (B 1-??) in appendix B.

With this set up, figures 8-10 show the transport terms $f_{\mathrm{Z}}$ as functions of $\tau$ for $\hat{\lambda}, \hat{\beta}=0$ and values $\hat{B}=0.5$ and 2 . The results for $f_{\Omega \mathrm{R}}$ (solid) in figure 8 may be compared with $f_{\mathrm{R}}$ (outer curve) in figure $2(\mathrm{a})$ : it is clear how increasing $\hat{B}$ gives rise to oscillations in these fluxes because of Alfvén waves. Likewise $f_{\mathrm{M}}$ in figure 10 may be compared with $f_{\mathrm{P}}$ for a passive scalar in figure 5(a) (outer curve).

We now begin to suffer from the presence of too many parameters in the problem 
(a)

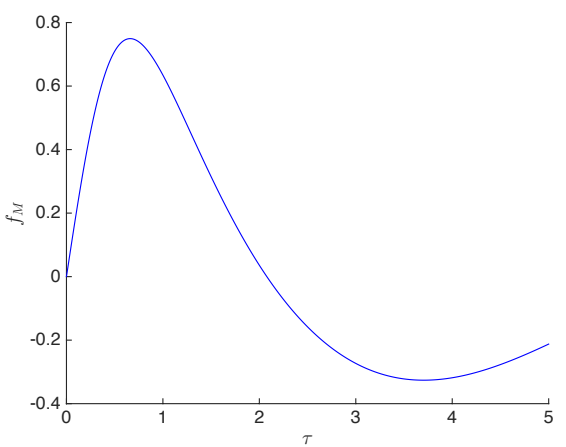

(b)

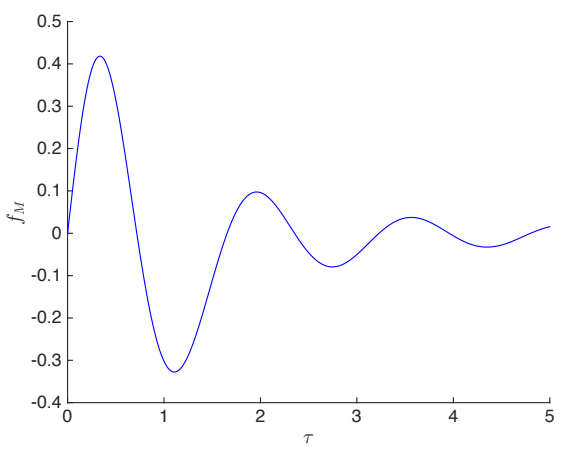

Figure 10. Plots of $f_{M}$ as a function of $\tau$ for $\hat{\lambda}=\hat{\beta}=0$ and (a) $\hat{B}=0.5$ and (b) $\hat{B}=2.0$.

(a)
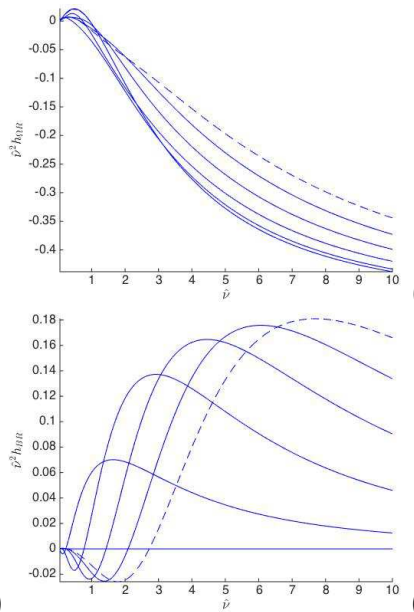

(d)

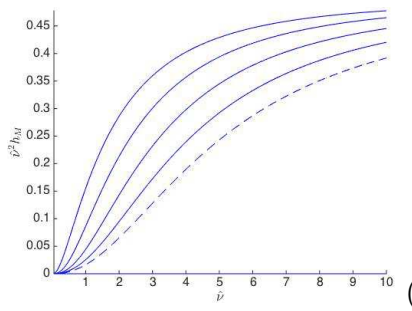

(b)

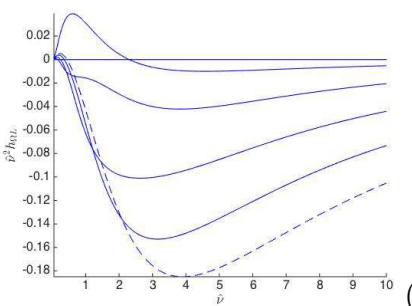

(c)
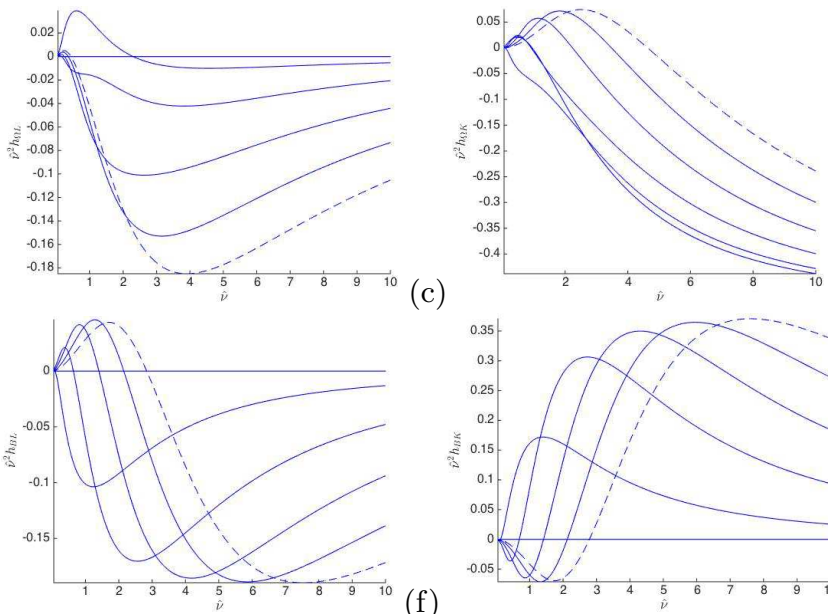

(e)

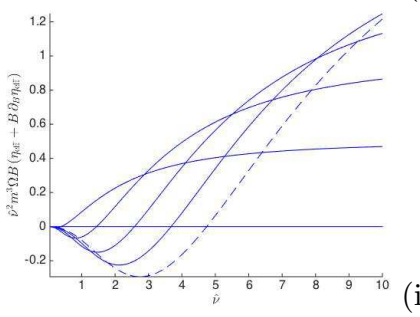

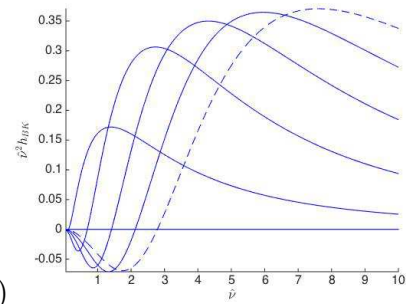

(f)

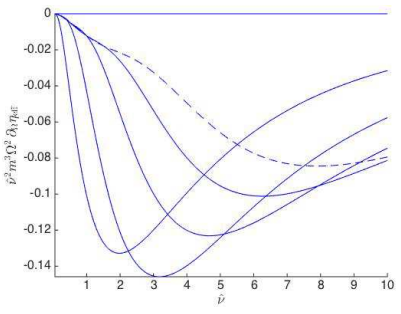

Figure 11. Plots of (a) $\hat{\lambda}^{2} h_{\Omega \mathrm{R}}$, (b) $\hat{\lambda}^{2} h_{\Omega \mathrm{L}}$, (c) $\hat{\lambda}^{2} h_{\Omega \mathrm{K}}$, (d) $\hat{\lambda}^{2} h_{B \mathrm{R}}$, (e) $\hat{\lambda}^{2} h_{B \mathrm{~L}}$, (f) $\hat{\lambda}^{2} h_{B \mathrm{~K}}$, (g) $\hat{\lambda}^{2} h_{\mathrm{M}}$, (h) $\hat{\lambda}^{2} m^{3} \Omega B\left(\eta_{\text {eff }}+B \partial_{B} \eta_{\text {eff }}\right)\left(\right.$ see (B 11)) and (i) $\hat{\lambda}^{2} m^{3} \Omega^{2} \partial_{\Omega} \eta_{\text {eff }}$ (see (B 12)), as functions of $\hat{\lambda}$ for $\hat{B}=0,1,2,3,4$ (solid) and 5 (dashed), with $\hat{\beta}=0$.

and struggle to plot the dependence of quantities $f_{\mathrm{Z}}(\tau, \hat{B}, \hat{\beta}, \hat{\lambda})$. However the quantities $\hat{\lambda}^{2} h_{\mathrm{Z}}(\hat{B}, \hat{\beta}, \hat{\lambda})$ (the prefactor chosen to show the clearest structure) are plotted as functions of $\hat{\lambda}$ for $\hat{\beta}=0$ and values of $\hat{B}$ in figure 11 . The values are $\hat{B}=0,1, \ldots 5$, with the last curve is dashed to mark it out. Where there are straight lines, this reflects the lack of coupling in the non-magnetic case $\hat{B}=0$. Some effects can clearly be seen, for example the effect of magnetic field is to suppress transport of $A$, via suppression of $\eta_{\text {eff }}$ or $h_{\mathrm{M}}$, depicted in figure $11(\mathrm{~g})$. These curves have been carefully confirmed against analytical 
approximations (see appendix B) for varying values of $\hat{B}, \hat{\lambda}, \hat{\beta} \gg 1$, as a partial check on our analysis and coding.

\subsection{Zonostrophic instability}

Although any calculations become somewhat opaque at this point, as we can only evaluate many quantities numerically, we can still press on to study zonostrophic instability for the MHD system, based on the large scale equations (5.34, 5.35). These are complicated as we have three effective transport terms, that themselves depend on the local vorticity $\Omega$ and magnetic field $B$.

We therefore think of the coefficients $\nu_{\text {eff }}, \chi_{\text {eff }}, \eta_{\text {eff }}$ as functions of $(\Omega, B, \beta, \lambda)$ and linearise $(5.34,5.35)$ about a state of constant vorticity $\Omega_{0}$ and field $B_{0}$. We thus replace

$$
\Omega \rightarrow \Omega_{0}+\Omega, \quad B \rightarrow B_{0}+B, \quad A \rightarrow B_{0} Y+A,
$$

where now $\Omega, B=A^{\prime}$ and $A$ are small quantities. We then obtain

$$
\begin{aligned}
& \partial_{\mathcal{T}} \Omega=\partial_{Y}\left[\left(\lambda+\nu_{\mathrm{eff}}\right) \partial_{Y} \Omega+\chi_{\mathrm{eff}} \partial_{Y} B\right], \\
& \partial_{\mathcal{T}} A=\partial_{Y}\left[\left(B_{0} \partial_{\Omega} \eta_{\mathrm{eff}}\right) \Omega+\left(\lambda+\eta_{\mathrm{eff}}+B_{0} \partial_{B} \eta_{\mathrm{eff}}\right) \partial_{Y} A\right],
\end{aligned}
$$

where the coefficients and their derivatives are evaluated at $(\Omega, B)=\left(\Omega_{0}, B_{0}\right)$. In general the equations are completely coupled, and if we seek a mode with $(\Omega, B)$ proportional to $\exp \left(P K^{2} \mathcal{T}+i K Y\right)$ we find growth rates $P$ satisfying

$$
\operatorname{det}\left[\left(\begin{array}{cc}
\lambda+\nu_{\mathrm{eff}} & \chi_{\mathrm{eff}} \\
B_{0} \partial_{\Omega} \eta_{\mathrm{eff}} & \lambda+\eta_{\mathrm{eff}}+B_{0} \partial_{B} \eta_{\mathrm{eff}}
\end{array}\right)+P \mathcal{I}\right]=0
$$

where $\mathcal{I}$ is the identity matrix.

We wish to investigate instabilities as a function of $\left(\Omega_{0}, B_{0}, \beta, \lambda\right)$, and we commence by setting $\beta=0$ and $\Omega_{0}=0$, no background shear. This is a major simplification, because for given $\lambda, B_{0}$, we use the limiting expressions for $\Omega_{0} \rightarrow 0$, that is for $\hat{\lambda}, \hat{B} \gg 1$. We are again effectively in a viscous regime (on the $\tau$ time scale) in which the exponential damping effect of the diffusion terms is dominant, for $\tau=O\left(\hat{\lambda}^{-1}\right) \ll 1$. On this time scale $\hat{B} \tau=O(1)$ also and so we need to use

$$
\hat{\zeta}_{0}=\cos \hat{B} \tau=\cos m B T, \quad \hat{\alpha}_{0}=i \sin \hat{B} \tau=i \sin m B T,
$$

as leading order approximations to solutions of (5.23) (see appendix B). (Note that a straightforward Maclaurin expansion in powers of $\tau$ is not satisfactory as this series becomes non-uniform when $\hat{B} \tau=O(1)$; our series is valid provided only $\hat{B} \tau^{2} \ll 1$.) This gives expressions for the $h_{\mathrm{Z}}$ which we do not set out here, but which have been used to check the numerical calculations in figure 11. More interestingly, we obtain

$\nu_{\mathrm{eff}}=-\frac{\lambda^{2}-m^{-2} B^{2}}{2 m^{6}\left(\lambda^{2}+m^{-2} B^{2}\right)^{2}}, \quad \chi_{\mathrm{eff}}=\Omega B \frac{3 \lambda^{2}-m^{-2} B^{2}}{m^{8}\left(\lambda^{2}+m^{-2} B^{2}\right)^{3}}, \quad \eta_{\mathrm{eff}}=\frac{1}{2 m^{6}\left(\lambda^{2}+m^{-2} B^{2}\right)}$,

valid for fixed $\lambda$ and $B$ with $\Omega \rightarrow 0$, at leading order.

Turning to (5.40), at $\Omega=\Omega_{0}=0$ both diagonal entries vanish, and in addition for the bottom right entry $\eta_{\text {eff }}+B \partial_{B} \eta_{\text {eff }}=-\nu_{\text {eff }}$ at this order. Thus we are left with explicit expressions for growth rates

$$
P=-\lambda-\nu_{\mathrm{eff}}, \quad P=-\lambda+\nu_{\mathrm{eff}} .
$$

The corresponding growth rate contours for $m^{2} P \geqslant 0$ are depicted in figure 12 . The lower set of contours, intersecting the horizontal axis, are for the first set of eigenvalues 
24

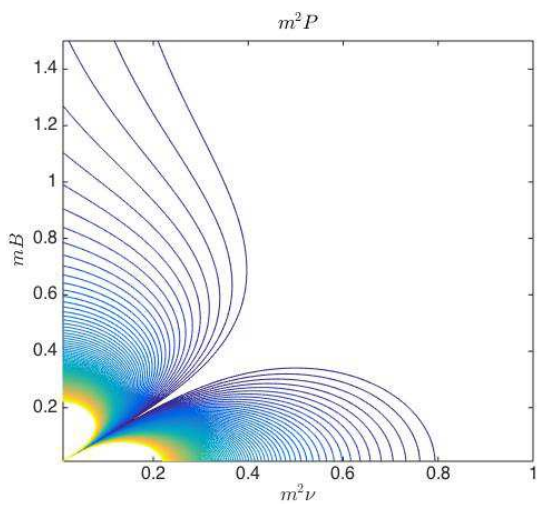

Figure 12. Contour plot of $m^{2} P(5.43)$ in the $\left(m^{2} \lambda, m B_{0}\right)$-plane for $\Omega_{0}=\beta=0$. Contours increase from $m^{2} P=0$ (outermost) in steps of 0.1 to $m^{2} P=10$.

in (5.43) and correspond to the hydrodynamic zonostrophic instability in section 3.4, modified by magnetic field. This has a suppressing effect, switching off the instability for $m^{2} B$ larger than about 0.34 , in at least qualitative agreement with results of Tobias, Hughes \& Diamond (2012)

The second set of eigenvalues in (5.43) gives contours intersecting the vertical axis in figure 12 . This is a magnetically driven instability, in which the variations of diffusivity $\eta_{\text {eff }}$ with $B$ lead to the tendency of the field to segregrate into regions of weaker and stronger $B$. The effect of removing field from regions of overturning motion is known as flux expulsion (Weiss 1966); in such regions the Lorentz force feedback is then reduced increasing the fluid flows and so enhancing flux expulsion, giving a runaway effect. The zonostrophic instability is restricted to a range of $\lambda$ values for $B_{0}=0$, whereas the magnetic flux expulsion instability occurs for any $B_{0}$ as $\lambda \rightarrow 0$. Note that this latter instability does not correspond to a sign change in $\chi_{\text {eff }}$, which would be impossible as the effective diffusivity arises from advection-diffusion of $A$, but is seen in (5.40) to emerge from the variation of $\chi_{\mathrm{eff}}$ with respect to changes in magnetic field $B$.

From this calculation we can now include a background shear, linearising about $\left(\Omega_{0}, B_{0}\right)$ with $\Omega_{0}>0$. As in the earlier section 3.4 we can no longer evaluate quantities $\nu_{\text {eff }}, \chi_{\text {eff }}$ and $\eta_{\text {eff }}$ analytically to obtain values of $P$ from (5.40), and so we compute them numerically. Figure 13 shows the contour plot of $m^{2} P$ for $(\mathrm{a}, \mathrm{b}) \Omega_{0}=0.1$ and $(\mathrm{c}, \mathrm{d}) 0.2$. The real parts of $m^{2} P$ are shown in panels $(\mathrm{a}, \mathrm{c})$ and these reveal that shear tends to suppress both types of instability. Interestingly though, there emerges near to the line $m B=m^{2} \lambda$ a region of oscillatory instability, with a pair of complex conjugate values of $m^{2} P$, whose imaginary parts are depicted in panels (b,d). This suggests emergence of a coupled instability through overstability. This can be seen to some extent in our analysis: in the equation (5.40), the product $\chi_{\text {eff }} B_{0} \partial_{\Omega} \eta_{\text {eff }}$ is negative (near $m B=m^{2} \lambda$ ) in our analytical approximation, from $(5.42, \mathrm{~B} 10)$. It also corresponds to the product of the quantities shown in figure 11(f,i) being negative, at least for small $B$. So, if the actual diagonal terms are small, the off-diagonal terms can give an imaginary part in $m^{2} P$. The oscillatory eigenvalue could be considered as resulting from a resonance between the two instabilities in figure 12.

The other effect we can include is that of a background vorticity gradient $\beta \neq 0$. We turn off the background shear, $\Omega_{0}=0$, and reintroduce the parameter $\hat{\beta}$. The corresponding transport coefficients may be computed, as we are again in the limit $\hat{\lambda}, \hat{\beta}, \hat{B} \gg 1$ as $\Omega_{0} \rightarrow 0$, and these are given in (B 14-B 16). The off-diagonal elements 
(a)
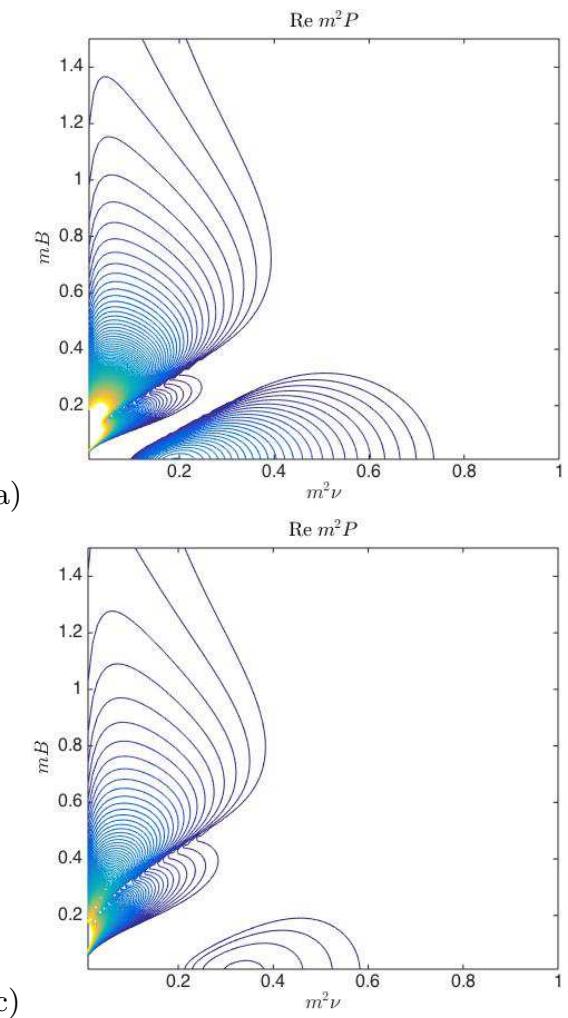

(b)

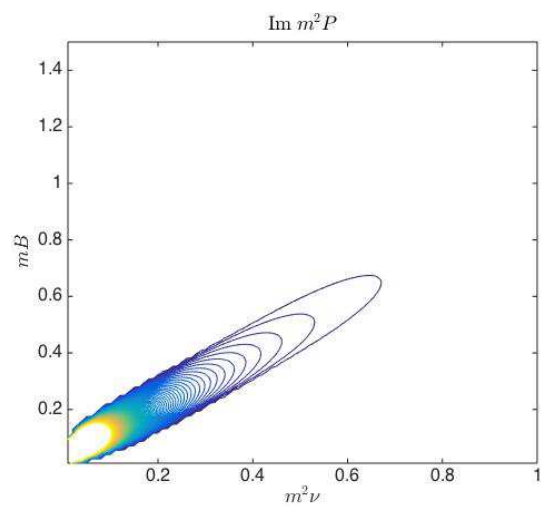

$\operatorname{Im} m^{2} P$

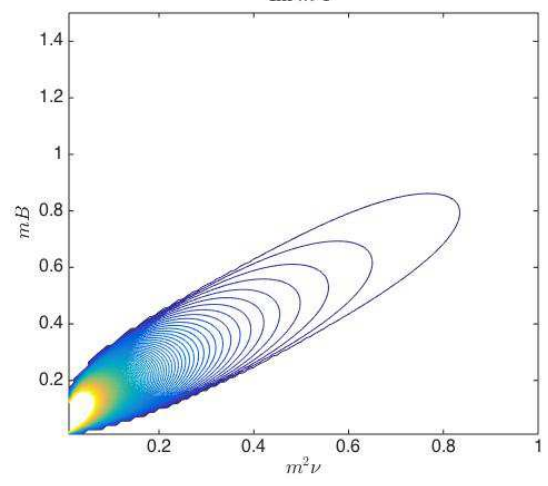

Figure 13. Contour plot of $m^{2} P$ from (5.40) in the $\left(m^{2} \lambda, m B_{0}\right)$-plane for $\beta=0$ and (a,b) $\Omega_{0}=0.1$, and (c,d) $\Omega_{0}=0.2$. In (a,c) the real part of $m^{2} P$ is shown, and in (b,d) the imaginary part. Contours increase from $m^{2} P=0$ (outermost) in steps of 0.1 to 10 .

are again zero (at this order) in the matrix (5.40) and so the problem decouples to eigenvalues given by the diagonal entries only. Figure 14 shows plots of the zonostrophic modes in $(\mathrm{a}, \mathrm{c})$ and the magnetic flux expulsion modes in $(\mathrm{b}, \mathrm{d})$ for $\beta=0.2$ and 0.4 . The effect of increasing $\beta$ is to suppress the magnetic instability, panels (b,d). However the zonostrophic instability contours now embrace the vertical axis. The combined effect of magnetic field $B$ and $\beta$ is to enhance zonostrophic instability, particularly for small viscosity. This can be contrasted with figure 12 for $\beta=0$, where the zonostrophic contours remain below the line $m^{2} \lambda=m B$. There is supporting evidence of enhanced instability in full numerical simulations (Durston 2015).

Finally we note that the effective terms $\nu_{\text {eff }}$ and $\chi_{\text {eff }}$ include contributions from both the Reynolds stress and Lorentz force terms. These contributions can be expressed as

$\lambda_{\text {eff R }}=-\frac{2 \lambda^{4}+m^{-2} \lambda^{2} B^{2}+m^{-4} B^{4}}{4 m^{6} \lambda^{2}\left(\lambda^{2}+m^{-2} B^{2}\right)^{2}}, \quad \lambda_{\text {eff } L}=-\frac{m^{-2} B^{2}\left(3 \lambda^{2}+m^{-2} B^{2}\right)}{4 m^{6} \lambda^{2}\left(\lambda^{2}+m^{-2} B^{2}\right)^{2}}, \quad \chi_{\text {eff R }}=-\chi_{\text {eff L }}=\frac{1}{2} \chi_{\mathrm{e}}$

Thus whereas two terms contribute equally and additively to $\chi_{\text {eff }}$, for the effective viscosity there is a leading order cancellation for large magnetic field $B \gg m \lambda$. The effect of the Lorentz force in cancelling Reynolds stresses for strong magnetic fields and the overall suppression of transport for large $B$ evident in (5.42) is discussed in Leprovost \& Kim (2009). 
26

(a)

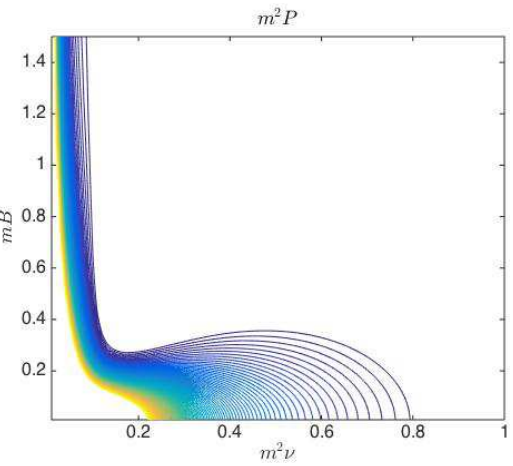

$m^{2} P$

(c)

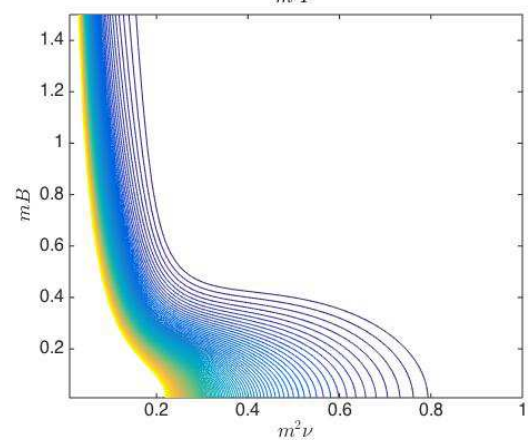

(b)

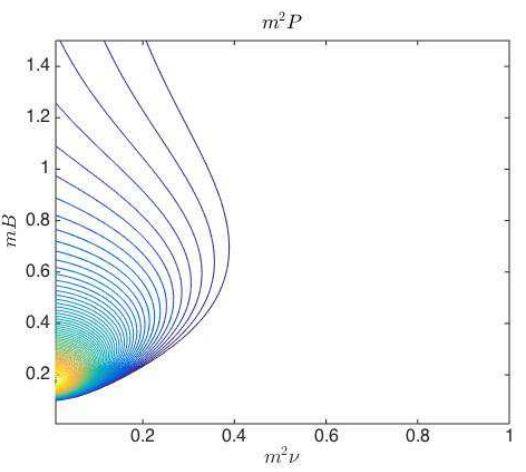

$m^{2} P$

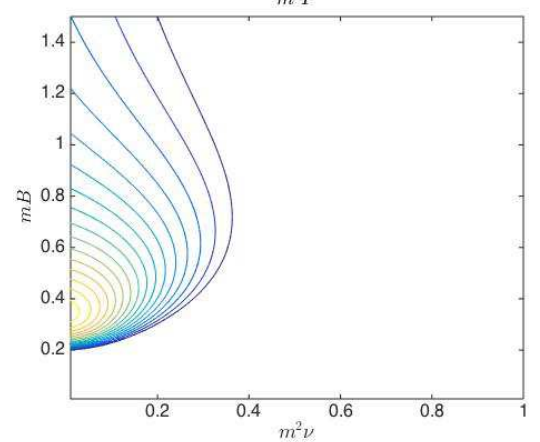

Figure 14. Contour plot of $m^{2} P$ (3.32) in the $\left(m^{2} \lambda, m B_{0}\right)$-plane for $\Omega_{0}=0$ and (a,b) $\beta=0.2$, and $(\mathrm{c}, \mathrm{d}) \beta=0.4$. In $(\mathrm{a}, \mathrm{c})$ contours for the zonostrophic instability are shown, and in $(\mathrm{b}, \mathrm{d})$ of the magnetic segregation instability. Contours increase from $m^{2} P=0$ (outermost) in steps of 0.1 to 10 .

\subsection{Equilibrium profiles}

Finally we consider steady equilibrium profiles in the full MHD system. For a steady profile we have

$$
\begin{aligned}
& m^{2}\left(\lambda+\nu_{\mathrm{eff}}\right) \partial_{Y} \Omega+m^{2} \chi_{\mathrm{eff}} \partial_{Y} B=C, \\
& m^{2}\left(\lambda+\eta_{\mathrm{eff}}\right) B=C_{B}, \\
& \partial_{Y} U=-\Omega, \quad \partial_{Y} A=B .
\end{aligned}
$$

In addition to $(3.35,4.12)$ we rescale

$$
B=C_{B} m^{-2} \lambda^{-1} \tilde{B}, \quad A=m^{2} \lambda C_{B} C^{-1} \tilde{A}, \quad \tilde{B}_{0}=m^{-3} \lambda^{-2} C_{B} ;
$$

here $A$ is analogous to the passive scalar in (4.12). Making use of (5.36), we obtain the equations

$$
\begin{aligned}
& {\left[1+\tilde{\lambda}^{-3} \tilde{\Omega}^{-2} h_{\Omega \mathrm{K}}\right] \partial_{\tilde{Y}} \tilde{\Omega}+\tilde{\lambda}^{-3} \tilde{\Omega}^{-1} \tilde{B}^{-1} h_{B \mathrm{~K}} \partial_{\tilde{Y}} \tilde{B}=1} \\
& {\left[1+\tilde{\lambda}^{-3} \tilde{\Omega}^{-2} h_{\mathrm{M}}\right] \tilde{B}=1} \\
& \partial_{\tilde{Y}} \tilde{U}=-\tilde{\Omega}, \quad \partial_{\tilde{Y}} \tilde{A}=\tilde{B}
\end{aligned}
$$

with now the functions

$$
h_{\mathrm{Z}}=h_{\mathrm{Z}}\left(\tilde{B}_{0} \tilde{B} \tilde{\Omega}^{-1}, \tilde{\beta} \tilde{\Omega}^{-1}, \tilde{\Omega}^{-1}\right)
$$

which we have to evaluate numerically. Note that here $\tilde{B}(Y)$ gives the magnetic field profile, but $\tilde{B}_{0}$ is a constant, a measure of the average field strength in the system. Given 
(a)
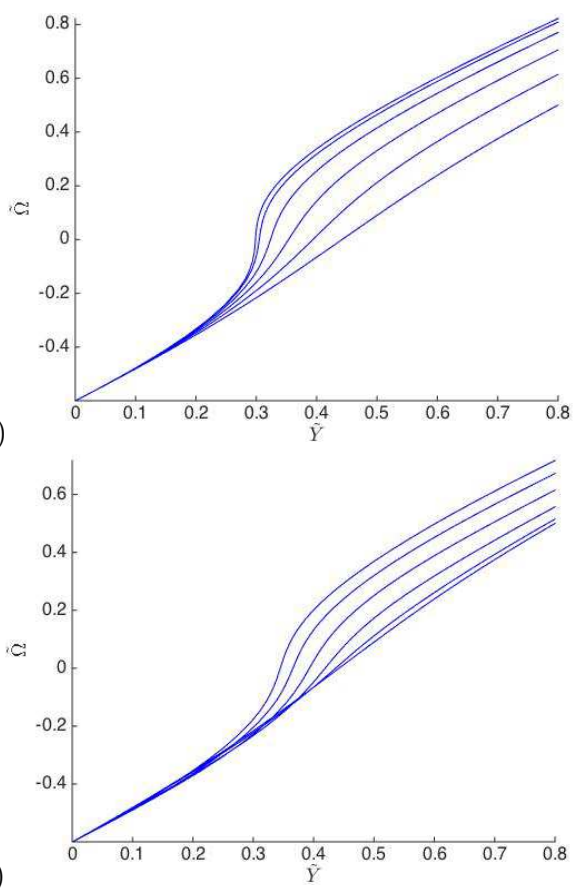

(b)
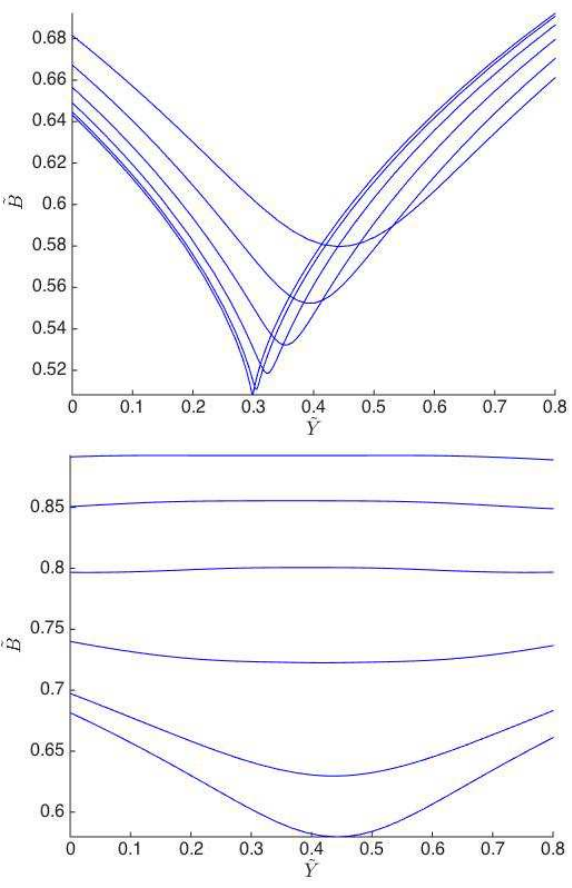

FIGURE 15. Plots of equilibrium profiles by integrating the ODE $(5.49-5.51)$ with $2-\tilde{\lambda}^{-3}=\frac{1}{16}$. Panel (a) shows $\tilde{\Omega}$ (reading down curves) and (b) $\tilde{B}$ (reading up on the left side) for $\tilde{\beta}=0$ and $\tilde{B}_{0}=0,0.2, \ldots, 1$. Panel (c) shows $\tilde{\Omega}$ (reading up) and (d) $\tilde{B}$ (reading up) for $\tilde{B}_{0}=1$ and $\tilde{\beta}=0,1, \ldots, 5$.

values for the parameters $\tilde{\lambda}, \tilde{\beta}$ and $\tilde{B}_{0}$, integrating the differential algebraic system (5.495.51) determines profiles for the fields.

We illustrate some profiles in figure 15 . Note that in the limit $\tilde{B}_{0}=0$, we regain the decoupled hydrodynamic and passive scalar problem, with profiles shown in figures $4(\mathrm{a})$ and $6(\mathrm{~b})$. With this in mind in figure $15(\mathrm{a}, \mathrm{b})$ we show profiles $\tilde{\Omega}(\tilde{Y})$ and $\tilde{B}(\tilde{Y})$ respectively where we start with $\tilde{B}_{0}=0$ and $\hat{\lambda}^{-3}=2-\frac{1}{16}$ (close to the threshold for zonostrophic instability). This gives the sharpest profiles for $\tilde{\Omega}$ and $\tilde{B}$ as in these earlier figures, and as the parameter $\tilde{B}_{0}$ is increased we observe smoother curves: the step in vorticity, and the effect of flux expulsion, are smoothed out in the presence of increasing magnetic fields. This could also be viewed as the effect of increasing magnetic field in moving the point in parameter space away from the threshold for zonostrophic instability (see figure 12), and indeed if $\hat{\lambda}$ is also adjusted, the profiles can be steepened again for non-zero magnetic field (not shown).

We continue to fix $\hat{\lambda}^{-3}=2-\frac{1}{16}$, take $\tilde{B}_{0}=1$ giving the two sharpest profiles in figure $15(\mathrm{a}, \mathrm{b})$, and then consider the effects of increasing $\tilde{\beta}$ in figure $15(\mathrm{c}, \mathrm{d})$. Interestingly the effect of increasing $\tilde{\beta}$ is to sharpen the vorticity profile again, while the magnetic field profile becomes smoother. The behaviour of the magnetic field profile is very similar to that for the analogous passive scalar gradient in figure 6(b). However the sharpening of the vorticity profile is not observed in the purely hydrodynamic system, and in that case there is actually no dependence on $\tilde{\beta}$ for vorticity profiles. So, we observe that the magnetic field has a catalysing effect: its presence allows the $\beta$-effect to sharpen the vorticity profile. This is in line with the earier discussion of zonostrophic instabilities: the 
combination of $\beta$-effect and magnetic field can be destabilising, leading to the increased areas of zonostrophic instability in figure $14(\mathrm{a}, \mathrm{c})$.

\section{Discussion}

In this paper we have considered forced two-dimensional flows in the presence of shear, $\beta$-effect, magnetic field and viscous damping. We have used a driving with a very basic structure in the interests of the analytical development, and discussed the hydrodynamic, passive scalar and magnetic field problems in turn. We have made contact with results elsewhere in the literature for hydrodynamic and passive scalar cases, though frequently other studies differ in significant aspects, for example in the type of dissipation or type of forcing. In particular the molecular dissipation used here is always Fickian diffusion, i.e. controlled by a Laplacian, $+\lambda \nabla^{2} \omega$; this brings in more subtle 'shear-diffuse' mechanisms than for the commonly used bottom drag $-\lambda_{0} \omega$, but complicates analysis (and leaves some mathematical questions unresolved, as indicated in appendix A). For future study, it would also be interesting to relax the requirement that all diffusivities are equal in $(2.7)$.

Key novel results we have obtained in the MHD problem are (i) quantifying the effect of the magnetic field in suppressing zonostrophic instability (discussed in Diamond et al. (2007) and observed numerically by Tobias, Hughes \& Diamond (2012)) and (ii) the presence of a further magnetic flux expulsion instability (figure 12), (iii) the suppression of these instabilities in the presence of shear (figure 13), (iv) the prediction of resonance leading to instability through over-stability (figure 14), and (v) that the combination of magnetic field and $\beta$-effect can be destablising in situations where neither individually drives an instability (figure 15). This latter effect is also identified in a study of transport by Keating \& Diamond (2008). We have also obtained a range of equilibrium profiles, representing steady state solutions in which the profile is governed by the effective transport coefficients, themselves nonlinear functions of the fields. These show vorticity steps and related features in passive scalar and magnetic fields.

In terms of the limitations and context of our work (and so directions for future study), clearly a major consideration is that it is based on the quasi-linear approximation, and while we have explored this in some depth, we have not in this paper attempted to verify predictions by full numerical simulations; see Tobias, Hughes \& Diamond (2012) and the preliminary results presented in Durston (2015). While there are several studies which show the utility of the quasi-linear approximation (e.g. Srinivasan \& Young 2012; Constantinou, Farrell \& Iouannou 2014) and while this approximation would certainly be valid in some parameter regimes (as per our scalings in $\varepsilon$ early in the paper), we recognise that the approach may principally be useful as a qualitative rather than quantitative guide to behaviour in full nonlinear simulations. We have also assumed scale separation between waves and mean flows, and this can be relaxed by using the correlation function formulation developed by Srinivasan \& Young (2012) and discussed by Bakas \& Iouannou (2013). This remains to be explored in the magnetic problem, together with the Ginzburg-Landau formulation of Parker \& Krommes (2013, 2014).

We also note that the forcing chosen is anisotropic and it is known that in the purely hydrodynamic problem the feedback on the mean flow is switched off completely in the case of an isotropic driving with $\beta=0$; this is explored in a number of papers, most recently Srinivasan \& Young (2014) who discuss the effects of a family of forcings going from isotropic to anisotropic. In our hydrodynamic set-up we also gain feedback at leading order in our expansions that arises through the anisotropy of the driving and which is independent of $\beta$. It would be interesting to rework the study for an isotropic driving and 
pick up the effect of $\beta$, known to be a term of the form $\beta^{2} \Omega^{\prime \prime \prime \prime}$ in the large scale vorticity equation (Srinivasan \& Young 2012; Bakas \& Iouannou 2013). This would involve taking our systematic expansion down two further orders, a significant task. With this, though, we note that the equilibrium profiles we obtain show only a single vorticity step (figure 4 ), and so tell us nothing about the important question of the natural spacing between steps in a periodic profile on a $\beta$-plane; perhaps bringing in a $\beta^{2} \Omega^{\prime \prime \prime \prime}$ term would give this information. Although one can argue about whether the driving in a 'real' fluid system would be isotropic or not, this is perhaps somewhat philosophical and more interesting would be to explore systems driven by convection (e.g. Morin \& Dormy 2004; Rotvig \& Jones 2006; Read et al. 2015) or some other natural instability, rather than an externally imposed body force. Finally we acknowledge that the study here is somewhat far from immediate application: perhaps the most relevant zone for the interaction of magnetic fields and waves in the $\beta$-plane setting is the solar tachocline (e.g. Hughes, Rosner \& Weiss 2007). The tachocline includes other physical processes, for example stratification, and its origin and persistence are not well understood, but nonetheless Diamond et al. (2007) argue that understanding of $\beta$-plane MHD is important for basic understanding of the interaction of forced Rossby and Alfven waves, and the present paper has aimed to elaborate such processes, albeit within the quasi-linear approximation.

\section{Acknowledgements}

We thank Eun-Jin Kim for references and valuable comments on an earlier draft, Steve Tobias for illuminating discussions and for sharing results with us prior to formal publication, and the referees for useful comments and references. We are grateful to the EPSRC for funding SD via a DTG research studentship.

\section{Appendix A. Local analysis near $\Omega=0$}

\section{A.1. Non-uniformity as $\lambda \rightarrow 0$}

In this appendix we pick up questions about the approximations we have used, at points where $\Omega$ goes to zero. We focus on the purely hydrodynamic formulation, where our starting point is the framework and exact solutions in section 3.3, but similar remarks would apply for the MHD case. Our analysis in section 3.3 is based on $Y, T, \lambda, \beta$ all being of order unity. With $\lambda>0$ taken of order unity, any wave is damped on a time scale of $T$, and so we do not care about the expansions perhaps becoming non-uniform beyond this point - there would be only a negligible contribution to fluxes. With this, we note that references to the 'inviscid' limit in the text above must be understood in these terms: one fixes $\lambda>0$ to a value, however small, and then the results are valid for that fixed value, as $\varepsilon \rightarrow 0$, i.e. in the limit of large scale separation.

To confirm this, and to see what happens when we try to relax these conditions, consider the key approximation, from (3.10),

$$
-\mathcal{L} \phi=m^{2}\left(1+\Omega^{2} T^{2}\right) \phi-i \varepsilon m \Omega^{\prime} T \phi-2 i \varepsilon m \Omega T \partial_{Y} \phi-\varepsilon^{2} \partial_{Y}^{2} \phi \simeq m^{2}\left(1+\Omega^{2} T^{2}\right) \phi .
$$

Clearly the main problem that could arise is at points, or in regions, where $\Omega=0$ (particularly given our scaling out of $\Omega$ in equation (3.22). However (with $T=O(1)$ the term $\varepsilon \Omega^{\prime} T \phi$ remains uniformly small compared with the RHS. More revealing is perhaps 
30

to substitute the leading order solution $\phi_{0}$ taken from (3.21) into the third term, with

$\varepsilon \Omega T \partial_{Y} \phi_{0}=\varepsilon \Omega T\left[\frac{-2 \Omega \Omega^{\prime} T^{2}}{1+\Omega^{2} T^{2}}-\frac{2}{3} m^{2} \lambda \Omega \Omega^{\prime} T^{3}+\frac{i \beta}{m}\left(-\frac{\Omega^{\prime}}{\Omega^{2}} \tan ^{-1} \Omega T+\frac{\Omega^{\prime} T}{\Omega\left(1+\Omega^{2} T^{2}\right)}\right)\right] \phi_{0}$.

As $\Omega$ tends to zero with $T=O(1)$ this remains uniformly small with respect to the RHS of (A 1). This is reassuring but not unexpected.

More interestingly, suppose we imagine now reducing $\lambda$, being interested in the inviscid limit for a fixed scale separation $\varepsilon \ll 1$. In fact let us set $\lambda=0$, and consider the approximation (A 1). For definiteness consider a locally quadratic profile

$$
U(Y)=\alpha Y^{2}, \quad \Omega(Y)=-2 \alpha Y,
$$

i.e. a generic zero-crossing of the vorticity profile. First take the term $\varepsilon \Omega^{\prime} T \phi_{0}$ in (A 1): this becomes of comparable size to the RHS when

$$
Y \sim \varepsilon \text { and } T \sim \varepsilon^{-1} .
$$

We can now revisit the terms in (A 2). The first term on the RHS of (A 2) also becomes comparable to the RHS of (A 1) when (A 4) holds. Other terms in (A 2) involve $\lambda$ and $\beta$, and so we imagine increasing these from zero: given the scalings (A 4 ) these terms begin to become comparable with the RHS of (A 1) when

$$
\lambda \sim \varepsilon \text { and } \beta \sim \varepsilon .
$$

To summarise, we have developed theory for $\lambda=O(1)(\lambda>0)$ and $\varepsilon \rightarrow 0$. If we wish to gain a uniform approximation which includes $\lambda=0$ for $\varepsilon \rightarrow 0$, then we need to investigate regions of non-uniformity given by (A 4). At the same time we could bring in $\lambda$ and $\beta$ with the appropriate scalings being (A 5 ).

\section{A.2. Long-time expansion}

We now develop some theory based on these new scalings. It is convenient to return to the original equations (2.11) with (2.14) (and no magnetic field or scalar transport). We replace

$$
\beta \rightarrow \varepsilon^{2} \beta, \quad \lambda \rightarrow \varepsilon^{2} \lambda,
$$

corresponding to (A 5). Looking at (A 4) we see that this corresponds to using $(y, \mathrm{~T})$ coordinates with $y=O(1)$ (the original $y$ ) and $\mathrm{T}=\varepsilon^{2} t$. The quadratic profile (A 3) becomes

$$
U(y)=\varepsilon^{2} \alpha y^{2}, \quad \Omega(y)=-2 \varepsilon^{2} y,
$$

with $\alpha$ a constant; in this section $\alpha, a, b, f, g, h$ are recycled and have no connection with these quantities in earlier sections. With these replacements the governing equations are simply

$$
\partial_{\top} \omega+i m \alpha y^{2} \omega=i m \tilde{\beta} \psi+\lambda\left(-m^{2}+\partial_{y}^{2}\right) \omega, \quad \omega=-\left(\partial_{y}^{2}-m^{2}\right) \psi
$$

with $\tilde{\beta}=\beta-2 \alpha$. This is the full inner problem, and may be solved numerically (Durston 2015).

For further analytical progress, though, we approximate $-m^{2}+\partial_{y}^{2} \simeq \partial_{y}^{2}$ for large gradients (as we will discuss later), giving

$$
\partial_{\top} \omega+i m \alpha y^{2} \omega=i m \tilde{\beta} \psi+\lambda \partial_{y}^{2} \omega, \quad \omega=-\partial_{y}^{2} \psi .
$$

Following Bajer, Bassom \& Gilbert (2001) we seek a solution of the form

$$
\omega=g(\mathrm{~T}) M(a+1, b, s), \quad \psi=h(\mathrm{~T}) M(a, b, s), \quad s=-i y^{2} f(\mathrm{~T}),
$$


where the $M$ are Kummer functions (see the NIST handbook, Olver et al. 2010) with parameters $a, b$ and argument $s$. The second of equations (A 9) is satisfied provided (NIST 13.2.1, 13.3.17)

$$
g=4 i a f h, \quad b=\frac{1}{2}
$$

The value of $b$ indicates that we could instead express what follows in terms of parabolic cylinder functions. The first of (A 9) becomes

$$
\begin{gathered}
\partial_{\mathrm{\top}} g M(a+1, b, s)+g f^{-1} \partial_{\mathrm{\top}} f s M^{\prime}(a+1, b, s)-m \alpha g f^{-1} s M(a+1, b, s) \\
=i m \tilde{\beta} h M(a, b, s)-4 i \lambda(a+1) f g M(a+2, b, s) .
\end{gathered}
$$

Now we express (NIST 13.3.17, 13.3.19),

$$
\begin{aligned}
& M(a, b, s)=\left[1+(a+1-b)^{-1} s\right] M(a+1, b, s)-(a+1-b)^{-1} s M^{\prime}(a+1, b, s), \\
& M(a+2, b, s)=M(a+1, b, s)+(a+1)^{-1} s M^{\prime}(a+1, b, s),
\end{aligned}
$$

for the terms on the right-hand side of (A 9) and gather terms in $M, s M$ and $s M^{\prime}$, all taken as functions of $(a+1, b, s)$. This yields three equations which may be simplified using (A 11) to give, respectively

$$
\begin{aligned}
& g^{-1} \partial_{\top} g=\frac{1}{4} m \tilde{\beta} a^{-1} f^{-1}-4 i \lambda(a+1) f, \\
& 4 a\left(a+\frac{1}{2}\right)=-\tilde{\beta} \alpha^{-1} \\
& \partial_{\top} f=m \alpha-4 i \lambda f^{2} .
\end{aligned}
$$

The middle of these fixes values of $a$ with

$$
4 a=-1 \pm \sqrt{1-4 \tilde{\beta} / \alpha}
$$

and we have (taking $\alpha>0$ for definiteness),

$$
f=m \alpha \mu^{-1} \tanh \mu \mathrm{\top}, \quad \mu=(1+i) \sqrt{2 \lambda m \alpha},
$$

and then

$$
\begin{aligned}
& g=c\left(\mu^{-1} \sinh \mu \mathrm{T}\right)^{-a-1 / 2}(\cosh \mu \mathrm{T})^{-a-1}, \\
& h=d\left(\mu^{-1} \sinh \mu \mathrm{T}\right)^{-a-3 / 2}(\cosh \mu \mathrm{T})^{-a}, \quad d / c=i\left(a+\frac{1}{2}\right) / m \tilde{\beta} .
\end{aligned}
$$

We thus obtain exact solutions of the PDEs (A 9) describing the evolution of waves subject to the flow, $\beta$-effect and viscosity. Note that for small viscosity $\mu \ll 1$ and $\mathcal{T} \ll \mu^{-1}$ (before viscosity acts), we have $f \simeq m \alpha \mathrm{T}$. Using the large- $s$ asymptotics for $M$, namely (NIST 13.7.2),

$$
M(a, b, s)=\frac{e^{s} s^{a-b}}{\Gamma(a)}+\frac{e^{ \pm i \pi a} s^{-a}}{\Gamma(b-a)}
$$

we obtain for large $s$

$$
\omega \propto y^{2 a+1} e^{i m \alpha y^{2} \mathrm{~T}}, \quad \psi \propto y^{2 a-1} \mathrm{~T}^{-1} e^{i m \alpha y^{2} \mathrm{\top}} .
$$

This should then be matched with the outer solutions in section 3.3, though it may be that the terms neglected in going from (A 8) to (A 9) have to be reincorporated. Note that in the case $\tilde{\beta}=0$, when the $\beta$ effect precisely cancels out the vorticity gradient from the flow, $\omega$ evolves like a passive scalar, and the root $a=-\frac{1}{2}$ correctly gives $|\omega|^{2}$ constant in (A 23). We leave this topic for further investigation, the aim being to obtain a complete (and useful) description of the evolution of waves and feedback outlined in section 3.3, valid for good scale separation $\varepsilon \ll 1$ uniformly in $\lambda \geqslant 0$. 


\section{Appendix B. Magnetohydrodynamic formulation}

In this appendix we give some of the less interesting calculations related to the MHD problem in section 5. First we use (5.23) to obtain a fourth order real system written symbolically as

$$
\left(\partial_{T}-\mathcal{M}\right) \mathcal{V}=0
$$

where

$\mathcal{V}(T, \Omega, B, \beta)=\left(\begin{array}{c}\left|\hat{\zeta}_{0}\right|^{2} \\ \left|\hat{\alpha}_{0}\right|^{2} \\ i\left(\hat{\zeta}_{0}^{*} \hat{\alpha}_{0}-\hat{\zeta}_{0} \hat{\alpha}_{0}^{*}\right) \\ \hat{\zeta}_{0}^{*} \hat{\alpha}_{0}+\hat{\zeta}_{0} \hat{\alpha}_{0}^{*}\end{array}\right), \quad \mathcal{M}(\tau, B, \beta)=\left(\begin{array}{cccc}0 & 0 & m B \mathcal{S}^{-1} & 0 \\ 0 & 0 & -m B \mathcal{S} & 0 \\ -2 m B \mathcal{S} & 2 m B \mathcal{S}^{-1} & 0 & m^{-1} \beta \mathcal{S} \\ 0 & 0 & -m^{-1} \beta \mathcal{S} & 0\end{array}\right)$,

$\mathcal{S}=\left(1+\tau^{2}\right)^{-1}$ and $\tau=\Omega T$ as usual. We think of $\mathcal{V}=\mathcal{V}(T, \Omega, B, \beta)$ and differentiating gives supplementary ODEs for $B \partial_{B} \mathcal{V}$ and $\Omega \partial_{\Omega} \mathcal{V}$,

$$
\begin{aligned}
& \left(\partial_{T}-\mathcal{M}\right)\left(B \partial_{B} \mathcal{V}\right)=\left(B \partial_{B} \mathcal{M}\right) \mathcal{V}, \\
& \left(\partial_{T}-\mathcal{M}\right)\left(\Omega \partial_{\Omega} \mathcal{V}\right)=\left(\tau \partial_{\tau} \mathcal{M}\right) \mathcal{V},
\end{aligned}
$$

which may be written, after a change of variable to $\tau$, as

$$
\begin{aligned}
& \left(\partial_{\tau}-\Omega^{-1} \mathcal{M}\right)\left(B \partial_{B} \mathcal{V}\right)=\left(\Omega^{-1} B \partial_{B} \mathcal{M}\right) \mathcal{V} \\
& \left(\partial_{\tau}-\Omega^{-1} \mathcal{M}\right)\left(\Omega \partial_{\Omega} \mathcal{V}\right)=\left(\Omega^{-1} \tau \partial_{\tau} \mathcal{M}\right) \mathcal{V} .
\end{aligned}
$$

To calculate the fluxes $f_{\mathrm{Z}}$ in $(5.28-5.32)$ numerically, we integrate the ODEs (B 1) together with (B 5), (B 6) in terms of $\tau$ for initial conditions $\mathcal{V}=(1,0,0,0)$, independent of $\Omega$ and $B$. We then extract any information needed at time $\tau$, for example for the figures $8-10$. The resulting numerical procedures were checked by computing derivatives numerically, and against analytical approximations below. Note that quantities such as $h_{\mathrm{Z}}$ were computed by incorporating in the system further ODEs of the form $d h_{\mathrm{Z}}^{\dagger} / d \tau=D^{2} f_{\mathrm{Z}}$ with $h_{\mathrm{Z}}^{\dagger}(0)=0$ and evaluating $h_{\mathrm{Z}}=h_{\mathrm{Z}}^{\dagger}(\tau)$ for $\tau$ large enough that $D^{2}$ is sufficiently small.

We now turn to the calculations involving instabilities in section 5.4 and outline parts omitted from the main text. First equations (5.23) may be expanded as

$$
\partial_{\tau} \hat{\zeta}_{0}=i \hat{B}\left(1+\tau^{2}\right) \hat{\alpha}_{0}+i \hat{\beta}\left(1-\tau^{2}+\tau^{4}-\cdots\right) \hat{\zeta}_{0} \quad \partial_{\tau} \hat{\alpha}_{0}=i \hat{B}\left(1-\tau^{2}+\tau^{4}-\cdots\right) \hat{\zeta}_{0},
$$

and then solutions developed perturbatively by bringing in successively higher powers of $\tau$. This gives, for $\hat{\beta}=0$,

$$
\begin{aligned}
& \hat{\zeta}_{0}=\cos \hat{B} \tau+\frac{1}{2} \hat{B}^{-2}\left[\hat{B}^{2} \tau^{2} \cos \hat{B} \tau-\hat{B} \tau \sin \hat{B} \tau\right]+\cdots, \\
& \hat{\alpha}_{0}=i \sin \hat{B} \tau+\frac{1}{2} i \hat{B}^{-2}\left[\left(1-\hat{B}^{2} \tau^{2}\right) \sin \hat{B} \tau-\hat{B} \tau \cos \hat{B} \tau\right]+\cdots .
\end{aligned}
$$

The leading order terms have been presented in (5.41) and give rise to the formulae (5.42) in due course; we omit the details. The second order terms are interesting as they determine $\eta_{\text {eff }}$ to better accuracy, namely

$$
\eta_{\mathrm{eff}}=\frac{1}{2 m^{6}\left(\lambda^{2}+m^{-2} B^{2}\right)}-\Omega^{2} \frac{8 \lambda^{6}+\lambda^{4} m^{-2} B^{2}+2 \lambda^{2} m^{-4} B^{4}+m^{-6} B^{6}}{4 m^{10} \lambda^{2}\left(\lambda^{2}+m^{-2} B^{2}\right)^{4}},
$$

and so give values for $B \partial_{\Omega} \eta_{\text {eff }}$, which otherwise vanishes at leading order.

Note that in the numerical calculations leading to figure $13(\mathrm{~b}, \mathrm{c})$, we differentiate $\eta_{\text {eff }}$ analytically before computing (5.40), with evaluation of the following integrals 
numerically,

$$
\begin{aligned}
& \eta_{\mathrm{eff}}+B \partial_{B} \eta_{\mathrm{eff}}=m^{-3} \Omega^{-1} B^{-1} \int_{0}^{\infty}-D^{2}\left(1+\tau^{2}\right)^{-1} B \partial_{B} i\left(\hat{\zeta}_{0}^{*} \hat{\alpha}_{0}-\hat{\zeta}_{0} \hat{\alpha}_{0}^{*}\right) d \tau, \\
& B \partial_{\Omega} \eta_{\mathrm{eff}}=m^{-3} \Omega^{-2} \int_{0}^{\infty} D^{2}\left(1+\tau^{2}\right)^{-1}\left[2 \tau^{2}\left(1+\tau^{2}\right)^{-1}+\frac{4}{3} \hat{\lambda} \tau^{3}-\Omega \partial_{\Omega}\right] i\left(\hat{\zeta}_{0}^{*} \hat{\alpha}_{0}-\hat{\zeta}_{0} \hat{\alpha}_{0}^{*}\right) d \tau .
\end{aligned}
$$

For $\hat{\beta} \neq 0$ solutions to (B 7) (just at leading order) are

$$
\left(\begin{array}{c}
\hat{\zeta}_{0} \\
\hat{\alpha}_{0}
\end{array}\right)=\frac{1}{p_{+}-p_{-}}\left[\left(\begin{array}{c}
p_{+} \\
\hat{B}
\end{array}\right) e^{i p_{+} \tau}-\left(\begin{array}{c}
p_{-} \\
\hat{B}
\end{array}\right) e^{i p_{-} \tau}\right], \quad p_{ \pm}=\frac{1}{2} \hat{\beta} \pm \sqrt{\frac{1}{4} \hat{\beta}^{2}+\hat{B}^{2}}
$$

(cf. Keating \& Diamond 2008) from which we can obtain

$$
\begin{aligned}
& \nu_{\mathrm{eff}}=-\frac{\lambda^{4}+\left(2-3 \Delta^{2}\right) \lambda^{2} \delta^{2}+\left(1-\Delta^{2}\right) \delta^{4}}{2 m^{6} \lambda^{2}\left(\lambda^{2}+\delta^{2}\right)^{2}} \\
& \chi_{\mathrm{eff}}=\Omega B \Delta^{2} \frac{3 \lambda^{2}-\delta^{2}}{m^{8}\left(\lambda^{2}+\delta^{2}\right)^{3}}+\Omega \beta^{2} \Delta^{4} \frac{\delta^{2}\left(3 \lambda^{2}+\delta^{2}\right)}{4 m^{10} B^{3} \lambda^{2}\left(\lambda^{2}+\delta^{2}\right)^{2}}, \\
& \eta_{\mathrm{eff}}=\frac{1}{2 m^{6}\left(\lambda^{2}+\delta^{2}\right)}, \quad \eta_{\mathrm{eff}}+B \partial_{B} \eta_{\mathrm{eff}}=\frac{\lambda^{2}+\left(1-2 \Delta^{2}\right) \delta^{2}}{2 m^{6}\left(\lambda^{2}+\delta^{2}\right)^{2}},
\end{aligned}
$$

where we have abbreviated (in this appendix only)

$$
\delta=\sqrt{m^{-2} B^{2}+\frac{1}{4} m^{-6} \beta^{2}}, \quad \Delta \delta=m^{-1} B .
$$

\section{REFERENCES}

Aubert, J. 2005 Steady zonal flows in spherical shell dynamos. J. Fluid Mech. 542, 53-67.

Bakas, N.A. \& Iouannou, P.J. 2011 Structural stability theory of two-dimensional fluid flow under stochastic forcing. J. Fluid Mech. 682, 332-361.

Bakas, N.A. \& Iouannou, P.J. 2013 On the mechanism underlying the spontaneous emergence of barotropic zonal jets. J. Atmos. Sci. 70, 2251-2271.

Bakas, N.A. \& Iouannou, P.J. 2014 A theory for the emergence of coherent structures in betaplane turbulence. J. Fluid Mech. 740, 312-341.

Bajer, K., Bassom, A.P. \& Gilbert, A.D. 2001 Accelerated diffusion in the centre of a vortex. $J$. Fluid Mech. 437, 395-411.

Bedrossian, J. \& Masmoudi, N. 2015 Inviscid damping and the asymptotic stability of planar shear flows in the 2D Euler equations. In Publications mathématiques de l'IHÉS, pp. 1-106. Springer.

Berloff, P., Kamenkovich, I. \& Pedlosky, J. 2009 A mechanism of multiple zonal jets in the oceans. J. Fluid Mech. 628, 395-425.

Bernoff, A.J. \& Lingevitch, J.F. 1994 Rapid relaxation of an axisymmetric vortex. Phys. Fluids 6, 3717-3723.

Bouchet, F. \& Morita, H. 2010 Large time behavior and asymptotic stability of the 2D Euler and linearized Euler equations. Physica D 239, 948-966.

Bouchet, F., Nardini, C. \& Tangarife, T. 2013 Kinetic theory of jet dynamics in the stochastic barotropic and 2d Navier-Stokes equations. J. Stat. Phys. 153, 572-625.

Bouchet, F., Nardini, C. \& Tangarife, T. 2014 Stochastic averaging, large deviations and random transitions for the dynamics of 2D and geostrophic turbulent vortices. Fluid. Dyn. Res. 46, 061416.

Chechkin, A.V. 1999 Negative magnetic viscosity in two dimensions. J.E.T.P. 89, 677-688.

Constantinou, N.C., Farrell, B.F. \& Iouannou, P.J. 2014 Emergence and equilibration of jets in beta-plane turbulence: applications of stochastic structural stability theory. J. Atmos. Sci. 71, 1818-1842. 
Diamond, P.H., Itoh, S.-I., Itoh, K. \& Silvers, L.J. 2007 Beta-plane MHD turbulence and dissipation in the solar tachocline. In The solar tachocline (ed. D.W. Hughes, R. Rosner \& N.O. Weiss). pp. 213-239. Cambridge University Press.

Dritschel, D.G. \& McIntyre, M.E. 2008 Multiple jets as PV staircases: the Phillips effect and the resilience of eddy-transport barriers. J. Atmos. Sci. 65, 855-874.

Dritschel, D.G \& Scott, R.K. 2011 Jet sharpening by turbulent mixing. Proc. R. Soc. Lond. A 369, 754-770.

Dunkerton, T.J. \& Scott, R.K. 2008 A barotropic model of the angular momentum conserving potential vorticity staircase in spherical geometry. J. Atmos. Sci. 65, 1105-1135.

Durston, S. 2015 Zonal jets and shear: transport properties of two-dimensional fluid flows. $\mathrm{PhD}$ thesis, University of Exeter, UK.

Farrell, B.F. \& Iouannou, P.J. 2003 Structural stability of turbulent jets. J. Atmos. Sci. 60, 2101-2118.

Farrell, B.F. \& Iouannou, P.J. 2006 Structure and spacing of jets in barotropic turbulence. J. Atmos. Sci. 64, 3652-3665.

Farrell, B.F. \& Iouannou, P.J. 2008 Formation of jets by baroclinic turbulence. J. Atmos. Sci. 65, 3353-3375.

Frisch, U., Legras, B. \& Villone, B. 1996 Large-scale Kolmogorov flow on the beta-plane and resonant wave interactions. Physica D 94, 36-56.

Galperin, B., Sukoriansky, S., Dikovskaya, N., Read, P.L., Yamazaki, Y.H. \& Wordsworth, R. 2006 Anisotropic turbulence and zonal jets in rotating flows with a $\beta$ effect. Nonlin. Proc. Geophys. 13, 83-98.

Galperin, B., Young, R.M.B., Sukoriansky, S., Dikovskaya, N., Read, P.L., Lancaster, A.J. \& Armstrong, D. 2014. Cassini observations reveal a regime of zonostrophic macroturbulence on Jupiter. Icarus 229, 295-320.

Heimpel, M. Aurnou, J. \& Wicht, J. 2005 Simulation of equatorial and high-latitude jets on Jupiter in a deep convection model. Nature 438, 193-196.

Hsu, P.-C. \& Diamond, P.H. 2015 Zonal flow formation in the presence of ambient mean shear. Phys. Plasmas 22, 022306.

Hughes, D.W., Rosner, R. \& Weiss, N.O. 2007 The solar tachocline. Cambridge University Press.

Keating, S.R. \& Diamond, P.H. 2008 Turbulent resistivity in wavy two-dimensional magnetohydrodynamic turbulence. J. Fluid Mech. 595, 173-202.

Kim, E.-J. 2007 The role of magnetic shear in flow shear suppression. Phys. Plasmas 18, 084504.

Kim, E.-J. \& MacGregor, K.B. 2003 Gravity wave driven flows in the solar tachocline: II stationary flows. Astrophys. J. 588, 645-654.

Leprovost, N. \& Kim, E.-J. 2008a Analytical theory of forced rotating sheared turbulence: the perpendicular case. Phys. Rev. E 78, 016301.

Leprovost, N. \& Kim, E.-J. 2008b Analytical theory of forced rotating sheared turbulence: the parallel case. Phys. Rev. E 78, 036319.

Leprovost, N. \& Kim, E.-J. 2009 Turbulent transport and dynamo in sheared MHD turbulence with a non-uniform magnetic field. Phys. Rev. E 80, 026302.

Manfroi, A.J. \& Young, W.R. 1998 Slow evolution of zonal jets on the beta plane. J. Atmos. Sci. 56, 784-800.

Manfroi, A.J. \& Young, W.R. 2002 Stability of $\beta$-plane Kolmogorov flow. Physica D 162, 208232.

Meshalkin, L.D. \& Sinai, I.G. 1961 Investigation of the stability of a stationary solution of a system of equations for the plane movement of an incompressible viscous fluid. Appl. Math. Mech. 25, 1700-1705.

Morin, V. \& Dormy, E. 2004 Time dependent $\beta$-convection in rapidly rotating spherical shells. Phys. Fluids 16, 1603-1609.

Newton, A., Kim, E.-J. \& Liu, H.-L. 2013 On the self-organizing process of large scale shear flows. Phys. Plasmas 20, 092306.

Olver, F.J.W., Lozier, D.W., Boisvert, R.F., \& Clark, C.W. 2010 NIST handbook of mathematical functions. Cambridge University Press.

Parker, J.B. \& Krommes, J.A. 2013 Zonal flow as pattern formation. Phys. Plasmas 20, 100703.

Parker, J.B. \& Krommes, J.A. 2014 Generation of zonal flows through symmetry breaking of statistical homogeneity. New J. Phys, 16, 035006. 
Read, P.L., Yamazaki, Y. H., Lewis, S.R., Williams, P.D., Wordsworth, R., Miki-Yamazaki, K., Sommeria, J., Didelle, H. \& Fincham, A.M. 2007 Dynamics of convectively driven banded jets in the laboratory. J. Atmos. Sci. 64, 4031-4052.

Read, P.L., Jacoby, T.N.L., Rogberg, P.H.T., Wordsworth, R.D., Yamazaki, Y.H., MikiYamazaki, K., Young, R.M.B., Sommeria, J., Didelle, H. \& Viboud, S. 2015 An experimental study of multiple zonal jet formation in rotating, thermally driven convective flows on a topographic beta-plane. Phys. Fluids 27, 085111.

Rhines, P.B. 1975 Waves and turbulence on the beta-plane. J. Fluid Mech. 69, 417-441.

Rotvig, J. \& Jones, C.A. 2006 Multiple jets and bursting in the rapidly rotating convecting twodimensional annulus model with nearly plane-parallel boundaries. J. Fluid Mech. 567, $117-140$.

Scott, R.K. \& Polvani, L.M. 2007 Forced-dissipative shalllow-water turbulence on the sphere and the atmospheric circulation of the giant planets. J. Atmos. Sci. 64, 3158-3176.

Scott, R.K. \& Dritschel, D.G. 2012 The structure of zonal jets in geostrophic turbulence. J. Fluid Mech. 711, 576-598.

Srinivasan, K. \& Young, W.R. 2012 Zonostrophic instability. J. Atmos. Sci. 69, 1633-1656.

Srinivasan, K. \& Young, W.R. 2014 Reynolds stress and eddy diffusivity of $\beta$-plane shear flows. J. Atmos. Sci. 71, 2169-2185.

Sukoriansky, S., Galperin, B. \& Chekhlov, A. 1999 Large scale drag representation in simulations of two-dimensional turbulence. Phys. Fluids 11, 3043-3053.

Tobias, S.M., Dagon, K.\& Marston, J.B. 2011 Astrophysical fluid dynamics via direct statistical simulation. Astrophys. J. 727, 127.

Tobias, S.M., Hughes, D.W. \& Diamond, P.H. $2007 \beta$-plane magnetohydrodynamic turbulence in the solar tachocline. Astrophys. J. 667, L113-116.

Vallis, G.K. \& Maltrud, M.E. 1993 Generation of mean flow and jets on a beta plane and over topography. J. Phys. Oceanogr. 23, 1346-1362.

Weiss, N.O. 1966 The expulsion of magnetic flux by eddies. Proc. R. Soc. Lond. A 293, 310-328.

Yadav, R.K., Gastine, T., Christensen, U.R. \& Reiners, A. 2015 Formation of starspots in selfconsistent global dynamo models: polar spots on cool stars. Astron. ES Astrophys. 573, A68.

Zhang, K. \& Jones, C.A. 1997 The effect of hyperviscosity on geodynamo models. Geophys. Res. Lett. 24, 2869-72.

Zheligovksy, V. 2011 Large-scale perturbations of magnetohydrodynamic regimes: linear and weakly nonlinear stability theory. Springer (Berlin). 